\title{
1 A three-wave network analysis of COVID-19's impact on schizotypal traits, paranoia and
}

2 mental health through loneliness

${ }^{1}$ Department of Psychology and Human Development, University College London, London, UK

$7 \quad{ }^{2}$ Neuropsychology and Applied Cognitive Neuroscience Laboratory, CAS Key Laboratory of

8 Mental Health, Institute of Psychology, Chinese Academy of Sciences, Beijing, China

$9 \quad{ }^{3}$ Department of Psychology, University of Chinese Academy of Sciences, Beijing, China

$10{ }^{4}$ Department of Psychology and Cognitive Science, University of Trento, Rovereto, Italy

115 Psychology Program, School of Social Sciences, Nanyang Technological University, Singapore

$12{ }^{6}$ Departments of Criminology, Psychiatry, and Psychology, University of Pennsylvania,

13 Philadelphia, USA

\section{Author Notes}

This manuscript has been submitted for publication and is likely to be edited as part of the peer- 


\section{Abstract (248/250)}

Background The 2019 coronavirus (COVID-19) pandemic has impacted people's mental wellbeing. Studies to date have examined the prevalence of mental health symptoms (anxiety, depression, loneliness), yet fewer longitudinal studies have compared across background factors and other psychological variables to identify vulnerable sub-groups. This study tests to what extent higher levels of psychotic-like experiences - indexed by schizotypal traits and paranoia are associated with various mental health variables 6- and 12-months since April 2020.

Methods Over 2,300 adult volunteers (18-89 years, female=74.9\%) with access to the study link online were recruited from the UK, USA, Greece, and Italy. Self-reported levels of schizotypy, paranoia, anxiety, depression, aggression, loneliness, and stress from three timepoints (17 April to 13 July 2020, $N_{l}=1,599 ; 17$ October to 31 January 2021, $N_{2}=774$; and 17 April to 31 July 2021, $N_{3}=586$ ) were mapped using network analysis and compared across time and background variables (sex, age, income, country).

Results Schizotypal traits and paranoia were positively associated with poorer mental health through loneliness, with no effect of age, sex, income levels, countries, and timepoints. Loneliness was the most influential variable across all networks, despite overall reductions in

52 levels of loneliness, schizotypy, paranoia, and aggression during the easing of lockdown.

53 Individuals with higher levels of schizotypal traits/paranoia reported poorer mental health

54 outcomes than individuals in the low-trait groups.

Conclusion Schizotypal traits and paranoia are associated with poor mental health outcomes

57 through self-perceived loneliness, suggesting that increasing social/community cohesion may

58 improve individuals' mental wellbeing in the long run.

Keywords: Network Analysis; Schizotypy; Anxiety; Depression; Stress; Loneliness; Sleep;

61 COVID-19; Longitudinal; Mental Health. 


\section{Introduction}

66 The coronavirus disease 2019 (COVID-19) pandemic has caused sustained global disruptions to

67 our livelihoods, yet the international scientific community has come together to collect timesensitive data to shape rapid government responses, policies, and vaccine development programs.

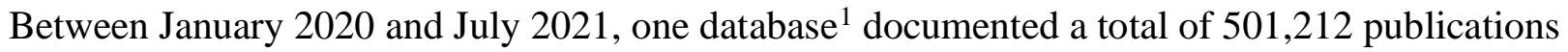
on coronavirus have been published, with mental health research being a key area of research interest. Some large birth cohort study findings reporting pre- and post-pandemic comparisons have been valuable in assessing change. Many more findings from newly developed crosssectional country-/population-specific studies have reported on mental health prevalence during

74 the early days of the pandemic. This latter set of studies has primarily defined mental health as 'internalizing' problems such as anxiety, depression, and loneliness (often excluding externalizing problems like aggression), focused on specific populations (e.g., medical frontline workers, teachers, parents with young children, children with special education needs) and often lacked a control group. While prevalence rates provide a good 'snapshot' of people's experiences during the pandemic, studies assessing the stability and change of these symptoms in the same individuals throughout the pandemic are limited due to COVID restrictions, with the exception of some timeseries studies. ${ }^{2}$ All in all, studies have aimed to examine possible environmental

82 factors, including the impact of national lockdown restrictions (e.g., physical distancing and

83 social isolation) on mental health (Carollo et al., 2021) in order to identify groups of individuals

84 who may be more vulnerable and in need of support.

Arguably a less researched yet important area is the impact of COVID-19 on psychotic-

87 that COVID-19 an airborne 'invisible killer' that has infected over 184 million people - many of

88 whom are asymptomatic - and caused 3.9 million deaths and counting globally, ${ }^{3}$ has instilled

89 doubt and distrust in all aspects of society. We know from existing research on paranoia, the

90 unfounded fixed belief that others cause intentional harm (Freeman \& Garety, 2000), that

\footnotetext{
${ }^{1}$ Dimensions COVID-19 database. https://reports.dimensions.ai/covid-19/

2 UCL COVID Social Study. https://www.covidsocialstudy.org/

${ }^{3}$ Data comes from Wikipedia, government health ministries, The New York Times, and other authoritative sources, as attributed.
} 
91 paranoia is a key symptom of schizophrenia-spectrum disorders like schizotypal personality

92 disorder - both of which exist in varying intensities in the general population (Bebbington et al.,

93 2013; Wong, \& Raine, 2018). For example, as of November 2020, 57\% of UK respondents aged

94 16-75 years $(N=2,244)$ expressed distrust in the government's control over the spread of

95 coronavirus, an increase from 28\% at the start of the pandemic in April 2020 (Ipsos MORI,

96 2020). Framing of public health messages which focus on the origin of coronavirus has caused

97 xenophobia towards people of Asian descent (Dhanani \& Franz, 2021). Fear of others not social

98 distancing, fear of catching COVID, lack of control over the restrictions, financial uncertainty,

99 are all well-documented stressors that may lead to heightened levels of suspicion towards others

100 and reclusive habits (Wong, 2020). It is conceivable then that lockdown will have a bigger effect

101 for individuals with higher levels of schizotypal traits and paranoia compared to their peers.

102 Compliance with government physical distancing and lockdown restrictions thought

103 necessary may perpetuate other health issues. For example, lockdown duration can likely

104 increase feelings of loneliness over the course of forced stay-at-home mandates (Carollo et al.,

105 2021) and fuel anxiety and psychotic-like experiences (Lim et al., 2018). Increased fear of one's

106 and others' safety, stress about COVID, and the lack of social contacts with others may fuel

107 maladaptive thoughts that if sustained may become paranoia known to be associated with poor

108 psychological wellbeing (Freeman et al., 2014); including, feelings of anxiety, worries (Freeman

109 et al., 2012), depression (Drake et al., 2014), insomnia (Freeman, Pugh, Vorontsova, \&

110 Southgate, 2009; Freeman et al., 2017), loneliness (Lamster et al., 2007) and to a lesser degree

111 aggression (Tone \& Davis, 2012; Wong, Freeman, \& Hughes, 2014). Psychotic-like experiences

112 as highlighted in a large representative sample of UK adults in April 2020, demonstrated that

113 mistrust and belief in conspiracy theories were associated with lower compliance in government

114 restrictions, antibody testing and vaccine adoption (Freeman et al., 2020). Thus, more than ever,

115 research on psychotic-like experiences and its correlates are of utmost importance in informing

116 public health and policy.

117 To the author's knowledge, four studies have investigated paranoia and schizotypal

118 personality traits in relation to mental health during the pandemic - although findings have been

119 mixed. In one study of UK and Germany adults between 27 April and 31 May 2020, 3.5\%

120 Germany) and 4.4\% (UK) respondents reported experiencing schizotypal traits for the first time 121 and a similar group reported increases in schizotypal traits after the pandemic (Germany $=4.1 \%$, 
122 UK $=4.8 \%)($ Knoelle, Ronan, \& Murray, 2021). By October 2020, researchers recruited an

123 additional sample and found an increase in schizotypal traits was associated with higher levels of

124 loneliness, use of drugs, and financial burden (Daimer et al., 2021). These changes were thought

125 to be due to national lockdown restrictions and physical distancing measures. In another cross-

126 sectional survey of Tunisian university students between 1 June and 15 July 2020, students in the

127 high schizotypal traits group (top-10\% on the 74-item Schizotypal Personality Questionnaire)

128 reported significantly more maladaptive coping strategies and fear of COVID-19 compared to

129 those in the low-schizotypy group (bottom-10\%) (Fekih-Romdhane, Dissem, \& Cheour, 2021).

130 Contrastingly, in an online survey of French adults between 13 April to 11 May $2020(N=728)$,

131 paranoia and hallucination were found to be relatively low and associated with cognitive-

132 affective experiences (loneliness, jumping-to-conclusions, anxiety, experiential avoidance), but 133 not associated with COVID19-related variables (e.g., length of isolation, hospitalisation, COVID

134 symptoms) (Bortolon et al., 2021). While these studies shed light on the mental health correlates

135 with schizotypal traits and paranoia during the pandemic, they are limited in the scope of mental

136 health variables and the short-term cross-sectional designs, which preclude the understanding of

137 specific target variable(s) for intervention as well as how relative associations change over time.

138 One way to fill these gaps is to use network analysis (NA). Mental health variables such

139 as anxiety, depression, aggression, and schizotypal traits are often correlated with each other, yet

140 traditional bivariate correlations only focus on the association between two variables each time

141 and preclude comparison across interactions or identification of the most influential variable in

142 the network across multiple time points. NA addresses this by estimating a network structure,

143 which consists of 'nodes' representing the variables and 'edges' representing the partial

144 correlations between each pair of variables (Borsboom \& Cramer, 2013; McNally, 2021; Wang

145 et al., 2020). Other common statistical comparisons include the 'centrality index' of nodes,

146 which reflect the influence of a node in the network and the 'strength' of the centrality indices,

147 which is the summed weight of all edges connected to a node in the network. By mapping the

148 nodes and estimating the edges, we can investigate the independent relationships between pairs

149 of variables whilst controlling for the effects of all the other variables and associations in the

150 network to obtain a more holistic view of the interactions between all the variables of interest as

151 a network and identify influential variables for intervention. 
This prospective study tests to what extent higher levels of psychotic-like experiences indexed by schizotypal traits and paranoia - relate with various mental health variables at 6- and

154 12-months since April 2020. Three 30-minute online surveys were conducted at three time155 points: 17 April to 13 July $2020\left(N_{l}=1,599\right), 17$ October to 31 January $2021\left(N_{2}=774\right)$ and 17 156 April to 31 July $2021\left(N_{3}=586\right)$ which coincide with the UK national lockdown 1 , lockdowns 2 157 and 3, and easing of restrictions respectively. It remains unclear how mental health variables 158 beyond internalizing problems, like externalizing problems (aggression), sleep quality, and

159 COVID-related stressors relate with schizotypal traits and paranoia over time during the 160 pandemic. Understanding how schizotypal traits and levels of paranoia have changed in relation 161 to both internalizing and externalizing problems for different groups of individuals (by sex, age, 162 income, country) during the pandemic can help inform government rapid response and COVID16319 recovery plans importantly, current public health interventions. Using a network analysis, this 164 study tests three hypotheses that:

165 1. Schizotypal traits and paranoia will be positively associated with both internalizing and externalizing problems.

2. The social networks may be the same or different for participants across different sex, age ( $<35$ vs $35+$ years), countries (UK vs Others), income level (low, medium, high), and timepoints (wave 1, 2, 3).

3. The network structure will be different for high vs low paranoid and schizotypal individuals, with associations being stronger for those in the high symptom groups.

177 the study, university lists, charity lists, Linkedln, Twitter, Instagram and word-of-mouth. All

178 adults aged 18 years and above with access to the study website www.GlobalCOVIDStudy.com 179 could take part. The 30-minute survey hosted online on Qualtrics was available in English and 7 180 other languages (Greek, Italian, Spanish, Chinese Traditional, Chinese Simplified, French, 181 German). Forward translations were first conducted by Google translate and cross-checked and 182 corrected by at least one native speaker. This study was pre-registered (https://osf.io/4nj3g/ on 17 183 April 2021) and ethical approval was obtained from the University College London Institute of 
184 Education Ethics and Review Committee in April 2020 (REC 1331; Wong \& Raine, 2020).

185 Informed consent was sought from participants at the start of the 30-minute online Qualtrics survey and at subsequent follow-ups, with opt-out options available throughout. Participant demographic and missing data on all study variables across the two waves of data collection are presented in Table 1. The analytic sample for this study consisted of data from participants at 3 time-points: wave $1\left(N_{l}=1599 ; 17\right.$ April to 14 July 2020), wave $2\left(N_{2}=774 ; 17\right.$ October 2020 to 31 January 2021), and wave $3\left(N_{3}=586 ; 17\right.$ April to 31 July 2021).

\subsection{Measures}

\subsubsection{Psychotic-like experiences (PLEs)}

Schizotypal traits were assessed by the Schizotypal Personality Questionnaire - Brief (SPQ-B; Raine \& Benishay, 1995), a 22-item yes/no questionnaire that when summed creates a total score ranging from 0 to 44 with a higher score reflecting more schizotypal traits. Three additional subscales were also created by summing the respective items to form the factors: Cognitive-Perceptual (F1), Interpersonal (F2), and Disorganized (F3) features of schizotypy. The internal reliability for the subscales and total score was good $(\alpha=.87)$.

Paranoia was assessed using the Social Mistrust Scale (SMS; Wong, Freeman, \& Hughes, 2014), a 12-item 3-point scale (No [0], Sometimes [1], Yes [2]). Summing all items created a

202 total mistrust score ranging from 0 to 24 , whereby a higher score reflected higher levels of

203 paranoia and suspiciousness. Past studies have denoted a score of 7 and above to be 'mistrustful'.

204 The internal reliability for the total score was $\operatorname{good}(\alpha=.79)$.

\subsubsection{Externalizing problems}

Self-reported levels of aggression were assessed by the Reactive-Proactive Questionnaire

208 (RPQ; Raine et al., 2006), a 23-item self-report questionnaire with a never (0), sometimes (1), 209 often (2) scale. Summing all items produces a total aggression score ranging from 0 to 46 with a 210 higher score reflecting more aggressive behaviours with good internal reliability $(\alpha=.85)$.

\subsubsection{Internalizing problems}

213 Depression was assessed using the Patient Health Questionnaire-9 (PHQ-9: Kroenke et 214 al., 2001) 9-item 4-point scale (not at all [0], several days [1], more than half the days [2], nearly 
215 every day [3]) which when summed produce a total score ranging from 0 to 27 . A higher score

216 reflected higher levels of depressive symptoms and a score above 15 was the clinical cut-off. The

217 internal reliability for this study was excellent $(\alpha=.90)$.

218 Anxiety was assessed using the General Anxiety Disorder-7 (GAD-7; Spitzer et al., 2006)

219 7-item 4-point scale (not at all [0], several days [1], more than half the days [2], nearly every day

220 [3]) where a higher summed score across the 7-items ranging from 0 to 21 reflects higher levels

221 of anxiety, with a score above 15 being the clinical cut-off. The internal reliability for this study

222 was excellent $(\alpha=.92)$.

223 The Loneliness Questionnaire (LQ; Russell, 1996) is a 20-item (10 reverse-coded items)

224 4-point scale (never [1], rarely [2], sometimes [2], often [3]) that when summed creates a total

225 score ranging from 20 to 77. A higher score denotes higher levels of loneliness. The internal

226 reliability for this study was excellent $(\alpha=.94)$.

\subsubsection{COVID-19-related stressors}

Participants selected from a list of 27 potential stressors related to the COVID-19

230 pandemic that they thought caused them stress in the past 14 days. Participants were shown a

231 follow-up question with the selected stressors and asked to what extent the following stressors

232 have caused them stress on a 5-point scale: No stress (0), A little bit of stress (1), Moderate

233 Stress (2), Quite a lot of stress (3), Extremely Stressful (4). Scores were summed and ranged

234 from 0 to 92.

\subsubsection{Sleep quality}

Self-reported sleep quality was indexed by summing 4-items from The Consensus Sleep

239 quality?', 'How would you rate the quality of your sleep overall?' and 'How rested or refreshed

240 do you feel when you wake up?') and the Karolinska Sleepiness Scale (Åkerstedt \& Gillberg,

241 1990), 'How sleepy have you felt during the last 5 minutes?'. Scores were summed and range

242 from 4 to 23 with moderate internal reliability $(\alpha=.66)$.

\subsubsection{Demographic variables}


Participants were asked to report on their date of birth $(<35$ or $35+)$, gender $($ female $=\%)$, 246 and country at the time of completing the survey (UK vs Other), which were dichotomized and

247 included in our between-group analyses (see Table 1).

\subsubsection{Covariates}

Participants reported on their annual pre-tax income in $\$ / £ 10,000$ bands (under $£ 30,000$ [0], £30,000-£59,999 [1], £60,000+ [2]), which was categorized and included in our analyses as 252 covariates.

\subsection{Data analysis}

The descriptive statistics of all study variables are reported in Table $1 \& 2$ and bivariate relationships are reported in Table 3.

Group comparison. Independent sample $t$ tests were performed to examine the differences between age groups (older vs. younger), gender groups or sites (UK vs. other counties). Paired sample t-tests were also performed to examine the changes of all psychological variables between two waves. SPSS 19.0 was used for descriptive analysis and t tests mentioned above, and a significant threshold was set as $p<0.05$.

Network Estimation. Firstly, psychological networks were estimated in whole sample collected at first wave to examine direct links between psychological variables including anxiety (GAD), depression (PHQ), sleep, COVID-related levels of stress, loneliness, aggressions (RPQ), social mistrust (SMS) and the three factors of the schizotypy subscales (SPQ-B). Nodes and edges are core components of a network. In this study, nodes were defined as participants' scores on psychological scales and edges were calculated using partial correlations between each pair of

268 nodes after controlling for all the other variables in the network. Graphical Least Absolute

269 Shrinkage and Selection Operator (LASSO) (Tibshirani, 1996) in combination with Extended

270 Bayesian Information Criteria (EBIC) model selection (Foygel \& Drton, 2010) were used to 271 estimate Gaussian graphical model and construct networks. In addition, the importance of each 272 node in the network was further investigated by examining the strength of each node by 273 summing up all connections of the node. Out of all the centrality indices, we mainly report the 274 index of "strength" as all connections are positive, and nodes are total or subscale scores of 275 psychological questionnaires. The standardized z scores of centrality indices were calculated and 
276 reported. The "bootnet" package (https://CRAN.R-project.org/package=bootnet) implemented in

277 R statistical software (version 4.0.2, https://www.r-project.org/) were used for network

278 construction and "qgraph" package (https://CRAN.Rproject.org/package=qgraph) was used for

279 centrality calculation and visualization. Force-directed Fruchterman-Reingold algorithm

280 (Fruchterman \& Reingold, 1991) was used to determine the placement of nodes in the network

281 and how they are estimated in the sample.

282 Network Comparison Test (NCT). The "Network Comparison Test" package

283 (https://CRAN.R-project.org/package=NetworkComparisonTest) was used to examine

284 invariance of two networks. The tests of network invariance usually include invariance of

285 network structure, global strength, and edge weights of the network. In order to compare the

286 networks between age groups, gender groups, countries as well as income levels, we estimated

287 networks for each subset of data and then performed the NCT respectively using two-tailed

288 permutation tests (10,000 times) (van Borkulo et al., 2017). In addition, to address multiple

289 comparisons of invariance tests of edge-weights and nodal strength, false discovery rate (FDR)

290 correction was used. The significance threshold was set at $p$ or adjusted $p<0.05$.

291 Network stability and accuracy. The stability and accuracy of each network we

292 estimated in this study were examined according to a tutorial paper (Epskamp et al., 2018) (see

293 Supplementary Figures S1-S8).

\section{3. Results}

\subsection{Descriptive statistics.}

297 Descriptive statistics of study variables (Table 1 and 2) and bivariate correlations of all study

298 variables are presented below (Table 3). All correlation coefficients were statistically significant 299 and positively correlated with each other at $p<0.001$ level.

301 Table 1. Demographic statistics of all study variables.

\begin{tabular}{cccc}
\hline Wave 1 & Wave 2 & Wave 3 \\
17 April to 14 July & 17 October 2020 to 31 & 17 April to 31 July 2021 \\
2020 & January 2021 & $\left(N_{3}=586\right)$ \\
$\left(N_{l}=1599\right)$ & $\left(N_{2}=774\right)$ & & n \\
\hline
\end{tabular}

Age 


$\begin{array}{ccccccc}<35 \text { years } & 952 & 59.5 & 446 & 57.6 & 339 & 57.8 \\ >=35 \text { years } & 642 & 40.2 & 323 & 41.7 & 244 & 41.6 \\ \text { Missing } & 5 & 0.3 & 5 & 0.6 & 3 & 0.5\end{array}$

\section{Gender}

Male

404

25.3

174

22.5

134

22.9

Female

1172

73.3

589

76.1

444

75.8

Else

23

1.4

11

1.4

8

1.4

\section{Countries}

UK

Others

Missing

Income

Low $(<30 \mathrm{k})$
Medium $(30-60 \mathrm{k})$
High $(>60 \mathrm{k})$
Missing

$649 \quad 40.6$

360

46.5

281

48

$576 \quad 36$

234

30.2

162

27.6

374

23.4

180

23.3

143

24.4

$639 \quad 40$

40

281

36.3

179

30.5

$348 \quad 21.8$

519

32.5

165

21.3

155

26.5

292

37.7

232

39.6

$93 \quad 5.8$

36

4.7

20

3.4

302

303 
304 Table 2. Descriptive statistics of all variables in network.

\begin{tabular}{|c|c|c|c|c|c|c|c|c|}
\hline Wave 1 & $n$ & range & $\min$. & $\max$ & $M$ & $S D$ & skewness & kurtosis \\
\hline SPQ-B Total & 1599 & 22 & 0 & 22 & 6.15 & 4.71 & 0.73 & -0.09 \\
\hline SPQ-B F1 & 1599 & 8 & 0 & 8 & 1.73 & 1.82 & 1.07 & 0.55 \\
\hline SPQ-B F2 & 1599 & 8 & 0 & 8 & 2.99 & 2.36 & 0.44 & -0.86 \\
\hline SPQ-B F3 & 1599 & 6 & 0 & 6 & 1.43 & 1.69 & 1.08 & 0.14 \\
\hline SMS Total & 1599 & 24 & 0 & 24 & 2.38 & 2.95 & 1.90 & 5.04 \\
\hline RPQ Total & 1599 & 34 & 0 & 34 & 6.74 & 4.56 & 1.04 & 2.02 \\
\hline PHQ-9 & 1599 & 27 & 0 & 27 & 7.29 & 5.60 & 0.94 & 0.44 \\
\hline GAD-7 & 1599 & 21 & 0 & 21 & 5.60 & 4.96 & 1.04 & 0.40 \\
\hline Stress Total & 1599 & 72 & 0 & 72 & 15.24 & 11.26 & 1.26 & 2.12 \\
\hline LQ Total & 1599 & 57 & 20 & 77 & 42.49 & 11.22 & 0.43 & -0.44 \\
\hline Sleep Total & 1599 & 19 & 4 & 23 & 12.42 & 3.69 & 0.08 & -0.57 \\
\hline Wave 2 & $n$ & range & $\min$. & $\max$. & $M$ & $S D$ & skewness & kurtosis \\
\hline SPQ-B total & 774 & 21 & 0 & 21 & 5.67 & 4.82 & 0.79 & -0.16 \\
\hline SPQ-B F1 & 774 & 8 & 0 & 8 & 1.50 & 1.78 & 1.25 & 1.04 \\
\hline SPQ-B F2 & 774 & 8 & 0 & 8 & 2.88 & 2.47 & 0.52 & -0.87 \\
\hline SPQ-B F3 & 774 & 6 & 0 & 6 & 1.29 & 1.64 & 1.20 & 0.43 \\
\hline SMS Total & 774 & 24 & 0 & 24 & 2.10 & 2.91 & 2.29 & 7.92 \\
\hline RPQ Total & 774 & 24 & 0 & 24 & 4.05 & 3.97 & 1.34 & 2.28 \\
\hline PHQ-9 & 774 & 27 & 0 & 27 & 7.14 & 5.80 & 1.03 & 0.58 \\
\hline GAD-7 & 774 & 21 & 0 & 21 & 5.56 & 5.00 & 1.08 & 0.55 \\
\hline Stress Total & 774 & 92 & 0 & 92 & 15.46 & 11.41 & 1.22 & 2.82 \\
\hline LQ Total & 774 & 57 & 20 & 77 & 42.77 & 11.72 & 0.41 & -0.51 \\
\hline Sleep Total & 774 & 18 & 4 & 22 & 13.03 & 3.67 & -0.07 & -0.59 \\
\hline Wave 3 & $n$ & range & $\min$. & $\max$. & $M$ & $S D$ & skewness & kurtosis \\
\hline SPQ-B Total & 586 & 22 & 0 & 22 & 5.35 & 4.64 & 0.95 & 0.39 \\
\hline SPQ-B F1 & 586 & 8 & 0 & 8 & 1.32 & 1.68 & 1.40 & 1.49 \\
\hline SPQ-B F2 & 586 & 8 & 0 & 8 & 2.83 & 2.45 & 0.57 & -0.76 \\
\hline SPQ-B F3 & 586 & 6 & 0 & 6 & 1.20 & 1.61 & 1.34 & 0.85 \\
\hline SMS Total & 586 & 24 & 0 & 24 & 1.90 & 2.88 & 2.58 & 9.59 \\
\hline RPQ Total & 586 & 30 & 0 & 30 & 3.60 & 3.92 & 2.02 & 6.56 \\
\hline PHQ-9 & 586 & 27 & 0 & 27 & 6.86 & 5.94 & 1.33 & 1.38 \\
\hline GAD-7 & 586 & 21 & 0 & 21 & 5.47 & 5.06 & 1.22 & 0.94 \\
\hline Stress Total & 586 & 59 & 0 & 59 & 12.95 & 10.57 & 1.54 & 2.54 \\
\hline LQ Total & 586 & 55 & 20 & 75 & 41.38 & 11.81 & 0.52 & -0.26 \\
\hline Sleep Total & 586 & 19 & 4 & 23 & 12.81 & 3.57 & 0.14 & -0.26 \\
\hline
\end{tabular}

305 Note. SPQ-B: Schizotypal Personality Questionnaire - Brief; SPQ-B F1: Cognitive-Perceptual; 306 SPQ-B F2: Interpersonal, SPQ-B F3: Disorganized; SMS: Social Mistrust Scale; RPQ: Reactive307 Proactive Questionnaire; PHQ-9: Patient Health Questionnaire-9; GAD-7: General Anxiety 308 Disorder-7; LQ: Loneliness Questionnaire. 
310 Table 3. Bivariate Pearson's correlation coefficients between study variables in the network at 311 wave 2.

\begin{tabular}{lccccccccccc}
\hline & $\mathbf{1}$ & $\mathbf{2}$ & $\mathbf{3}$ & $\mathbf{4}$ & $\mathbf{5}$ & $\mathbf{6}$ & $\mathbf{7}$ & $\mathbf{8}$ & $\mathbf{9}$ & $\mathbf{1 0}$ & $\mathbf{1 1}$ \\
\hline 1. SPQ-B Total & - & & & & & & & & & \\
2. SPQ-B F1 & .765 & - & & & & & & & & \\
3. SPQ-B F2 & .839 & .413 & - & & & & & & & \\
4. SPQ-B F3 & .792 & .479 & .494 & - & & & & & & \\
5. SMS Total & .453 & .403 & .336 & .358 & - & & & & & \\
6. RPQ Total & .335 & .360 & .193 & .276 & .311 & - & & & & \\
7. PHQ-9 & .426 & .347 & .350 & .324 & .392 & .278 & - & & & \\
8. GAD-7 & .420 & .396 & .319 & .298 & .354 & .336 & .752 & - & & \\
9. Stress Total & .270 & .272 & .203 & .177 & .283 & .256 & .565 & .595 & - & \\
10. LQ Total & .610 & .365 & .619 & .442 & .502 & .243 & .539 & .453 & .320 & - \\
11. Sleep Total & .240 & .187 & .204 & .182 & .238 & .137 & .558 & .454 & .352 & .338 & - \\
\hline
\end{tabular}

312 Notes. SPQ-B: Schizotypal Personality Questionnaire - Brief; SPQ-B F1: Cognitive-Perceptual;

313 SPQ-B F2: Interpersonal, SPQ-B F3: Disorganized; SMS: Social Mistrust Scale; RPQ: Reactive-

314 Proactive Questionnaire; PHQ: Patient Health Questionnaire-9; GAD: General Anxiety

315 Disorder-7; LQ: Loneliness Questionnaire.

\subsection{Comparisons of all study variables across age, gender, countries and income groups at} wave 1

319 Independent samples t-tests were performed to compare groups differences between younger and 320 older groups, males and females, as well as Countries (UK vs. Others). In addition, MANOVA 321 was conducted to compare groups with different levels of income. Adjusted $p(0.05 / 11=0.0045)$

322 was considered as a significance threshold to correct multiple comparisons. The results in detail 323 were shown in Table 4.

324 In summary, the younger group reported higher levels of schizotypal traits, aggression, 325 depression stress, and anxiety, as well as more sleep problems compared to older participants; 326 females reported more severe depression stress, and anxiety than male participants. Compared to 327 the other countries, participants from the UK had higher levels of schizotypal traits, depression, 328 anxiety, loneliness and sleep problems, and lower aggression. High income group showed a 
329 better situation in terms of schizotypal trait, negative affect, and loneliness compared to the other 330 two groups with medium- or low-income levels.

332 Table 4. Comparisons across age, gender, countries and income groups

\begin{tabular}{lccccccccc}
\hline & \multicolumn{2}{c}{ Age } & \multicolumn{2}{c}{ Gender } & \multicolumn{2}{c}{ Countries } & \multicolumn{3}{c}{ Levels of Income } \\
\multicolumn{1}{c}{ Wave 1 } & Younger vs. Older & \multicolumn{2}{c}{ Male vs. Female } & UK vs. others & \multicolumn{2}{c}{ (Low vs. Medium vs. High) } \\
\hline SPQ-B Total & $\mathbf{t}$ & $\mathrm{4}$ & $\mathrm{t}$ & $\mathrm{p}$ & $\mathrm{t}$ & $\mathrm{p}$ & $\mathrm{F}$ & $\mathrm{p}$ & Post hoc \\
SPQ-B F1 & $\mathbf{3 . 1 6}$ & 0.002 & -0.62 & 0.537 & 0.78 & 0.437 & $\mathbf{2 1 . 1 4}$ & $<0.001$ & $\mathrm{~L}>\mathrm{M}>\mathrm{H}$ \\
SPQ-B F2 & $\mathbf{3 . 0 9}$ & 0.002 & 1.06 & 0.289 & $\mathbf{3 . 5 0}$ & $<0.001$ & $\mathbf{1 8 . 8 7}$ & $<0.001$ & $\mathrm{~L}=\mathrm{M}>\mathrm{H}$ \\
SPQ-B F3 & $\mathbf{4 . 8 4}$ & $<0.001$ & $\mathbf{4 . 5 3}$ & $<0.001$ & 2.41 & 0.016 & $\mathbf{2 1 . 2 7}$ & $<0.001$ & $\mathrm{~L}>\mathrm{M}>\mathrm{H}$ \\
SMS Total & -1.28 & 0.201 & 1.51 & 0.131 & 0.40 & 0.691 & $\mathbf{2 9 . 1 5}$ & $<0.001$ & $\mathrm{~L}>\mathrm{M}>\mathrm{H}$ \\
RPQ Total & $\mathbf{3 . 2 2}$ & 0.001 & -0.69 & 0.493 & -2.84 & 0.005 & $\mathbf{2 1 . 9 6}$ & $<0.001$ & $\mathrm{~L}>\mathrm{M}=\mathrm{H}$ \\
PHQ-9 & $\mathbf{6 . 3 1}$ & $<0.001$ & $\mathbf{- 4 . 6 5}$ & $<0.001$ & $\mathbf{6 . 1 3}$ & $<0.001$ & $\mathbf{1 8 . 0 0}$ & $<0.001$ & $\mathrm{~L}=\mathrm{M}>\mathrm{H}$ \\
GAD-7 & $\mathbf{5 . 7 9}$ & $<0.001$ & $\mathbf{- 6 . 9 8}$ & $<0.001$ & $\mathbf{4 . 1 8}$ & $<0.001$ & $\mathbf{9 . 0 9}$ & $<0.001$ & $\mathrm{~L}=\mathrm{M}>\mathrm{H}$ \\
Stress Total & $\mathbf{5 . 7 1}$ & $<0.001$ & $\mathbf{- 5 . 0 0}$ & $<0.001$ & $\mathbf{3 . 0 0}$ & 0.003 & $\mathbf{1 6 . 2 0}$ & $<0.001$ & $\mathrm{~L}>\mathrm{M}>\mathrm{H}$ \\
LQ Total & 0.87 & 0.383 & 1.08 & 0.279 & $\mathbf{3 . 8 0}$ & $<0.001$ & $\mathbf{1 6 . 2 3}$ & $<0.001$ & $\mathrm{~L}=\mathrm{M}>\mathrm{H}$ \\
Sleep Total & $\mathbf{2 . 9 1}$ & 0.004 & -2.41 & 0.016 & $\mathbf{4 . 8 4}$ & $<0.001$ & 0.50 & 0.606 & -
\end{tabular}

333 Note. SPQ-B: Schizotypal Personality Questionnaire - Brief; SPQ-B F1: Cognitive-Perceptual;

334 SPQ-B F2: Interpersonal, SPQ-B F3: Disorganized; SMS: Social Mistrust Scale; RPQ: Reactive-

335 Proactive Questionnaire; PHQ: Patient Health Questionnaire-9; GAD: General Anxiety

336 Disorder-7; LQ: Loneliness Questionnaire. $\mathrm{p}<0.0045(0.05 / 11)$ was set as threshold to adjust for 337 multiple comparisons.

$340 \quad 3.3$ Comparisons of all study variables across time

341 To examine the changes across time, we conducted paired samples $t$ tests on all study variables

342 between Wave 1 and 2, as well as between Wave 2 and 3, respectively. The results suggested

343 that participants reported lower levels of aggression and more sleep problems at wave 2

344 compared to wave 1. At the last wave, participants had lower levels of schizotypal trait and stress

345 caused by COVID. These changes are significant after multiple comparison corrections with

346 adjusted $\mathrm{p}<0.0045$. 
348 Table 5. Comparisons of all study variables across time using paired samples $t$ tests

\begin{tabular}{lcccccccccc}
\hline & \multicolumn{4}{c}{ T1 vs. T2 } & \multicolumn{5}{c}{ T2 vs. T3 } \\
& mean diff. & $\boldsymbol{S} \boldsymbol{D}$ & $\boldsymbol{t}$ & $\boldsymbol{d} \boldsymbol{f}$ & $\boldsymbol{p}$ & mean diff. & $\boldsymbol{S D}$ & $\boldsymbol{t}$ & $\boldsymbol{d f}$ & $\boldsymbol{p}$ \\
\hline SPQ-B Total & 0.36 & 3.00 & $\mathbf{3 . 0 9}$ & 672 & 0.002 & 0.23 & 2.40 & 2.00 & 435 & 0.046 \\
SPQ-B F1 & 0.05 & 1.26 & 1.10 & 672 & 0.272 & 0.18 & 1.14 & $\mathbf{3 . 3 2}$ & 435 & 0.001 \\
SPQ-B F2 & 0.16 & 1.58 & 2.59 & 672 & 0.010 & -0.03 & 1.46 & -0.49 & 435 & 0.622 \\
SPQ-B F3 & 0.15 & 1.29 & $\mathbf{2 . 9 4}$ & 672 & 0.003 & 0.08 & 1.05 & 1.64 & 435 & 0.101 \\
SMS Total & 0.10 & 2.38 & 1.08 & 672 & 0.279 & 0.25 & 2.26 & 2.27 & 435 & 0.024 \\
RPQ Total & 2.42 & 3.89 & $\mathbf{1 6 . 1 7}$ & 672 & $<0.001$ & 0.37 & 3.20 & 2.38 & 435 & 0.018 \\
PHQ-9 & 0.15 & 4.33 & 0.87 & 672 & 0.383 & 0.16 & 4.30 & 0.77 & 435 & 0.443 \\
GAD-7 & -0.02 & 4.10 & -0.12 & 672 & 0.903 & -0.07 & 4.22 & -0.35 & 435 & 0.725 \\
Stress Total & 0.24 & 8.85 & 0.69 & 672 & 0.492 & 2.19 & 8.39 & $\mathbf{5 . 4 6}$ & 435 & $<0.001$ \\
LQ Total & -0.31 & 7.27 & -1.10 & 672 & 0.273 & 1.07 & 7.29 & $\mathbf{3 . 0 8}$ & 435 & 0.002 \\
Sleep Total & -0.56 & 3.53 & $\mathbf{- 4 . 1 3}$ & 672 & $<0.001$ & 0.20 & 3.16 & 1.29 & 435 & 0.199
\end{tabular}

349 Note. SPQ-B: Schizotypal Personality Questionnaire - Brief; SPQ-B F1: Cognitive-Perceptual;

350 SPQ-B F2: Interpersonal, SPQ-B F3: Disorganized; SMS: Social Mistrust Scale; RPQ: Reactive-

351 Proactive Questionnaire; PHQ: Patient Health Questionnaire-9; GAD: General Anxiety

352 Disorder-7; LQ: Loneliness Questionnaire. $\mathrm{p}<0.0045(0.05 / 11)$ was set as threshold to adjust for 353 multiple comparisons. 

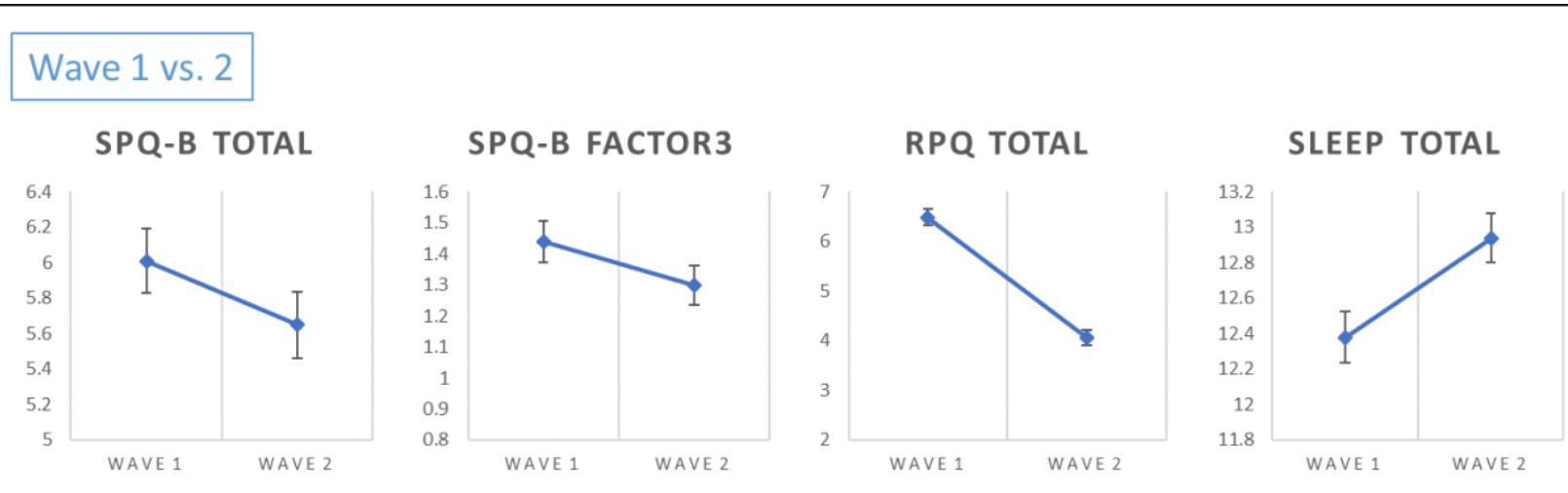

Wave 2 vs. 3

SPQ-B FACTOR1

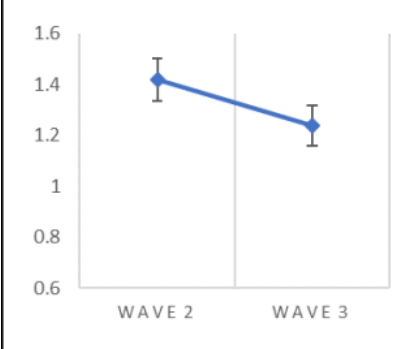

STRESS TOTAL

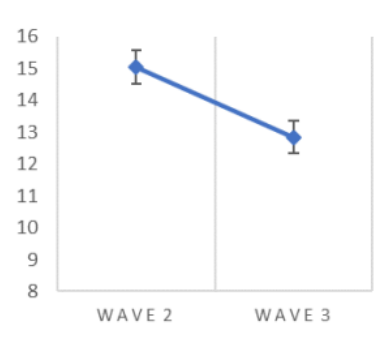

\subsection{Network analysis: network estimation and inference in the whole sample of wave 1}

In the whole sample of wave 1, we estimated a network using all study variables including three factors of the SPQ-B, shown in Figure 1. The line between a pair of variables indicates the partial correlations after controlling all the other variables in the network, thicker lines represent stronger connections. There are strong connections of schizotypal traits and social mistrust with mental health. For example, SPQ-B factor 1 was linked to anxiety and aggression, social mistrust was correlated with loneliness, aggression and depression. We also observed strong connections between the negative dimension of schizotypy, interpersonal deficits (SPQ-B F2) and loneliness.

The strength of all variables was shown in Figure 1, depression, anxiety and loneliness were the most influential nodes in the network as they had relatively high nodal strength. According to the network, anxiety, depression and stress from COVID were closely correlated with each other, while sleep problems were only linked to depression. More interestingly, we found that loneliness was connected with multiple nodes in the network, including schizotypal traits (SPQB F2 and F3), social mistrust and depression. This finding suggested that loneliness may serve as a bridge connecting both schizotypal traits/paranoia and mental health. 


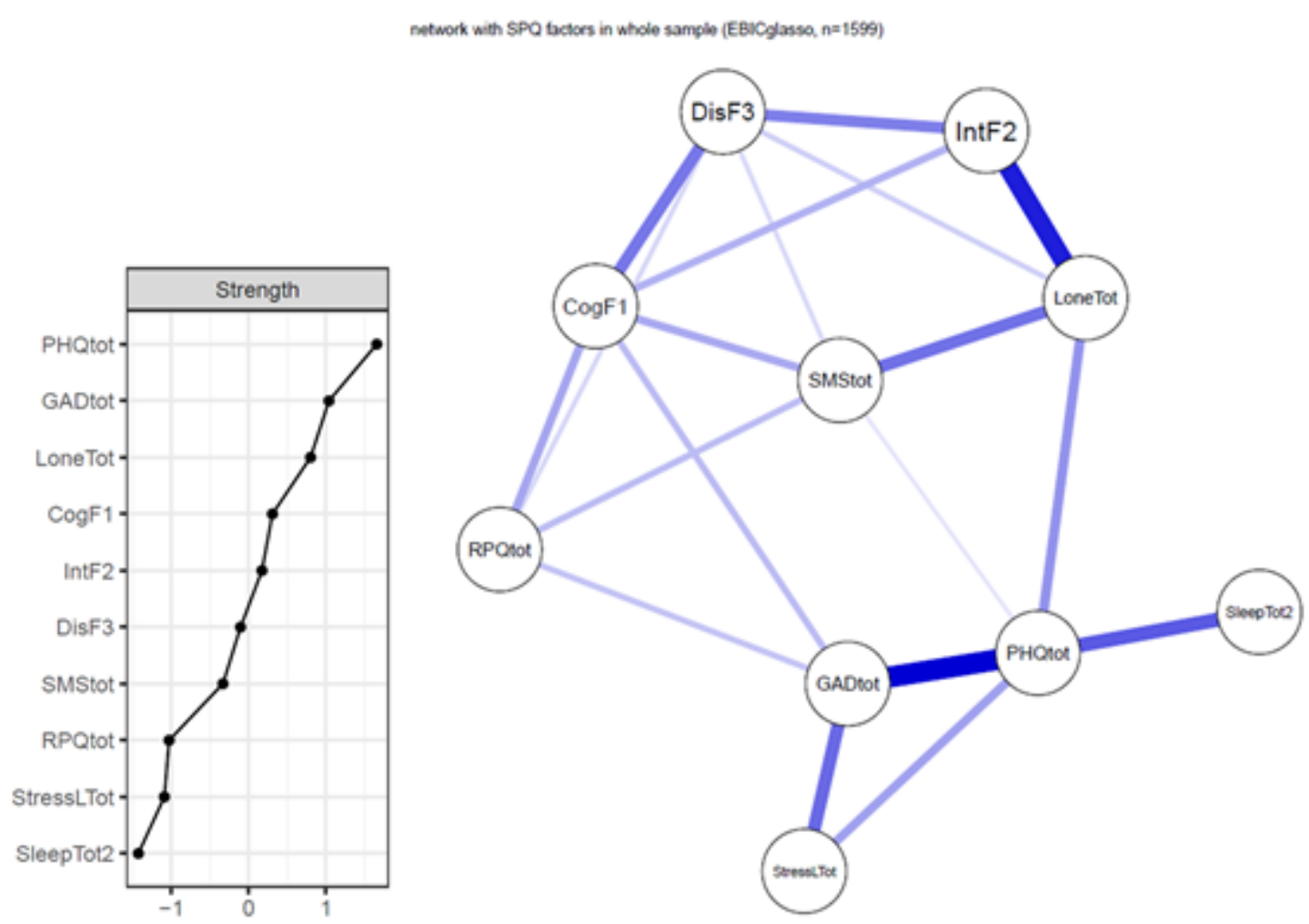

375 Figure 1. Estimated network structure using SPQ factor scores (right) and nodal strength

376 (left). All lines in the network showed positive partial correlations, thicker lines represent

377 stronger correlations. SPQ-B: Schizotypal Personality Questionnaire - Brief, SMStot: Social

378 Mistrust Scale, RPQtot: Reactive-Proactive Questionnaire, PHQtot: Patient Health

379 Questionnaire-9, GADtot: General Anxiety Disorder-7, LoneTot: Loneliness Questionnaire,

380 StressTot: COVID-19-related stressors, SleepTot: self-reported sleep quality, CogF1: Cognitive-

381 Perceptual factor of SPQ-B, IntF2: Interpersonal factor of SPQ-B, DisF3: Disorganized factor of 382 SPQ-B.

3.5 Network comparisons across groups

At the first wave, network comparisons were conducted across groups by age, gender, countries and levels of income.

The results of NCT did not show significant differences in terms of the invariance of network structures or global strength between age groups (younger vs. older groups, network structure invariance test: $M=0.12, p=0.243$; global strength invariance: 3.86 for younger group 
and 4.04 for older group, $S=0.18, p=0.106$, global strength for network of younger group is 3.86 and 4.04 for the network of older group). As sample sizes of two groups were different, we repeated NCT for 100 times using random subsamples of younger participants, only $1 \%$ and $16 \%$ of the invariance tests for network and global strength were found significant.

Similarly, we did not find any significant differences between male and female participants (network structure: $M=0.12, p=0.448$; global strength: $S=0.16, p=0.196$, global strength for the network of males is 3.86 and 4.02 for females). Repeated subsampling and NCT showed that only $13 \%$ and $3 \%$ in invariance tests of the network structure and global strength were significant respectively.

In addition, we compared the networks of participants from UK and other countries, no significant differences were found no matter on network structure $(M=0.15, p=0.170)$ or global

401 strength $(S=0.07, p=0.610$, global strength for the network of UK participants is 3.98 and 3.91 402 for others).

Among groups with low, medium and high levels of income, we performed a series of 404 NCT to compare the networks with each other and no significant differences were observed 405 (Low vs. Medium income group: network structure: $M=0.14, p=0.300$; global strength: $S=$ $4060.07, p=0.647$; Low vs. High income group: network structure: $M=0.13, p=0.335$; global 407 strength: $S=0.06, p=0.570$; Medium vs. High income group: network structure: $M=0.23, p<$ 408 0.05; global strength: $S=0.003, p=0.984$ ).

409 These findings indicated that networks were comparable across different groups 410 including age groups, gender groups, countries as well as groups with different levels of income.

\subsection{Network comparisons across three waves}

We also performed the network comparisons to test the invariance of network structure

414 and global strength across three waves with each other (Figure 2). Compared to the Wave 1

415 network, Wave 2 network had comparable network structure $(M=0.11, p=0.153)$ and global

416 strength $(S=0.02, p=0.879,3.99$ for wave 1 and 4.02 for wave 2$)$, suggesting that no

417 significant differences on the networks were found across two waves. Similarly, the networks of 418 Wave 2, and Wave 3 are similar as no significant differences were found $(M=0.08, p=0.983 ; S$ $419=0.07, p=0.519$, global strength is 4.02 for wave 2 and 3.95 for wave 3 ). These findings 
indicate that network structure and partial correlations among variables were similar across three 421 waves.

Figure 2. Invariance test of network structures across three time-points.

424
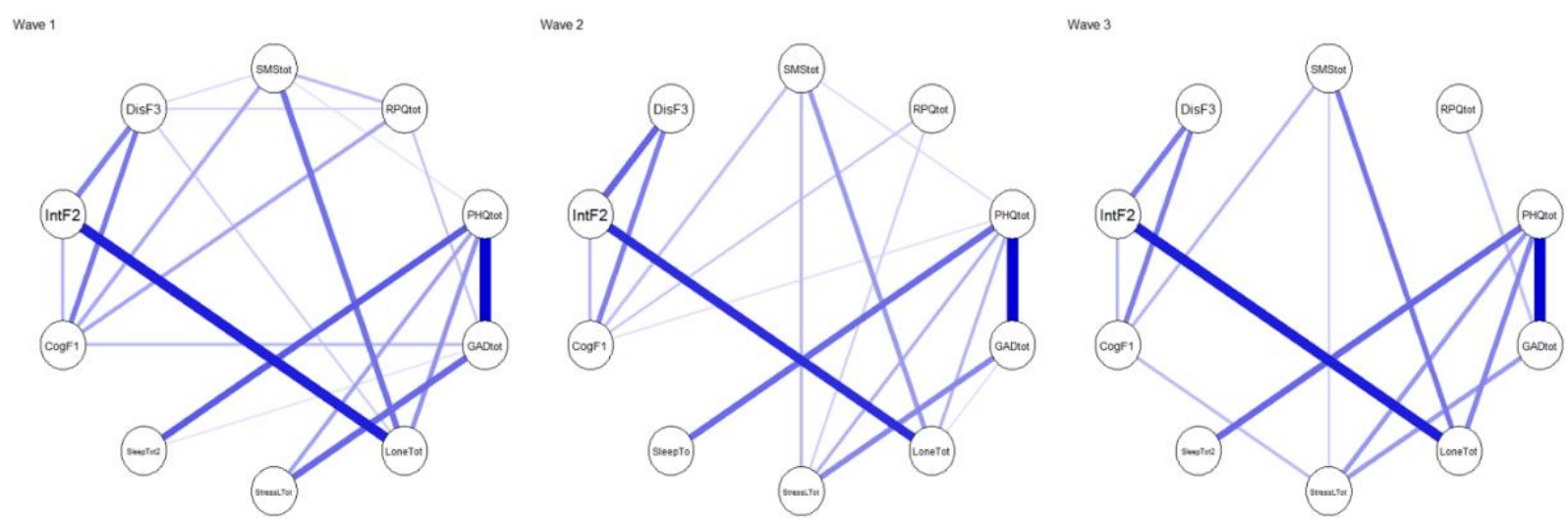

425

3.7. Network comparisons between high vs. low schizotypy/paranoia

The network structures between groups with high and low SPQ-B scores were different $(M=0.21, p<0.001)$. The individuals with high SPQ-B showed significantly stronger correlations between social mistrust and SPQ-B factor1(adjusted $p=0.005$ ), between anxiety and SPQ-B factor1 (adjusted $p=0.027$ ), as well as between loneliness and SPQ-B factor2 (adjusted $\mathrm{p}$ $<0.001)$. The global strength of the high schizotypy group is higher than the low schizotypy group $(S=1.10, p<0.001,2.66$ for low SPQ group and 3.76 for high SPQ group).

In terms of the social mistrust, the high SMS group also showed a different network structure from the low social mistrust group $(M=0.183, p=0.004)$. The connections of social mistrust with SPQ-B factor 1 (adjusted $p<0.05$ ) and loneliness (adjusted $p<0.001$ ) were stronger in the network of the high SMS group than the low SMS group. The global strength for the high SMS group is 3.82, significantly higher than the global strength of the low SMS group which is $3.30(\mathrm{~S}=0.53, p<0.05)$. Networks were shown in Figure 3.

Figure 3. Networks of all study variables by high-/low-schizotypy groups (top) and high-/lowsocial mistrust groups (bottom). 
High Schizotypy

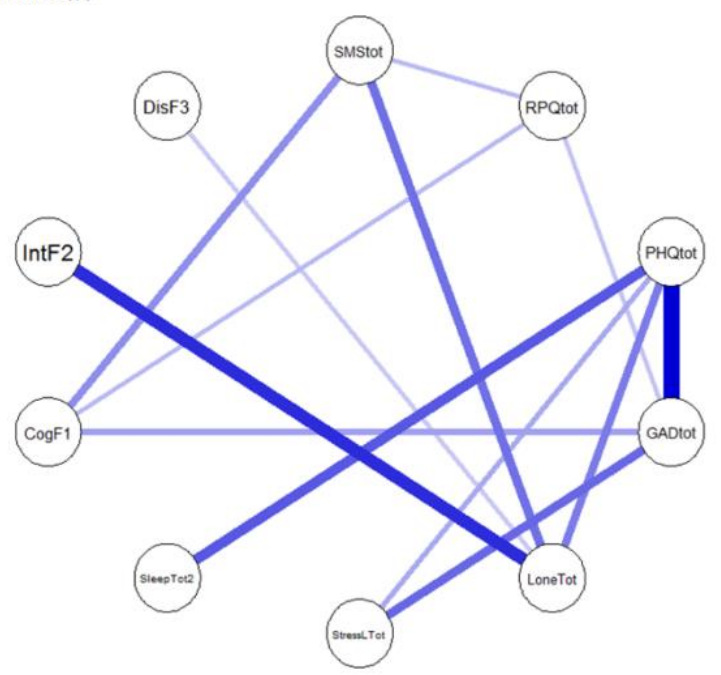

444

High Social Mistrust

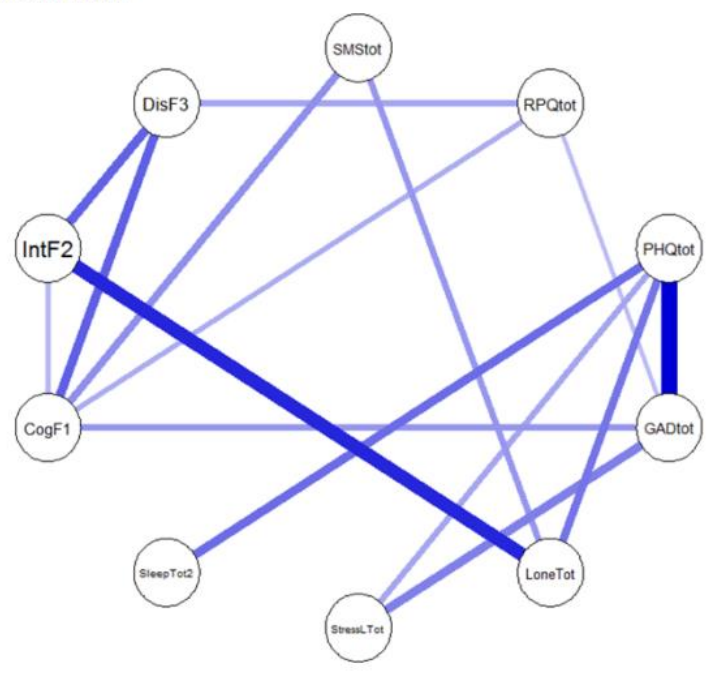

Low Schizotypy

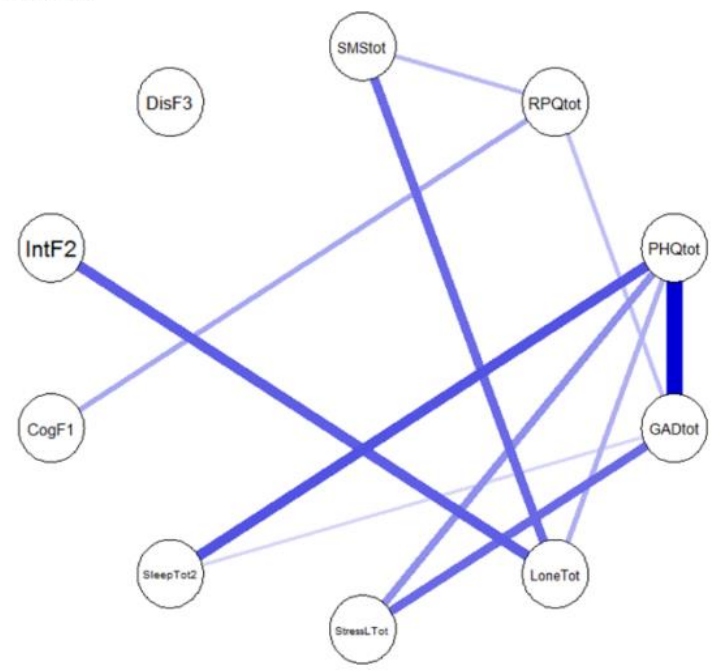

Low Social Mistrust

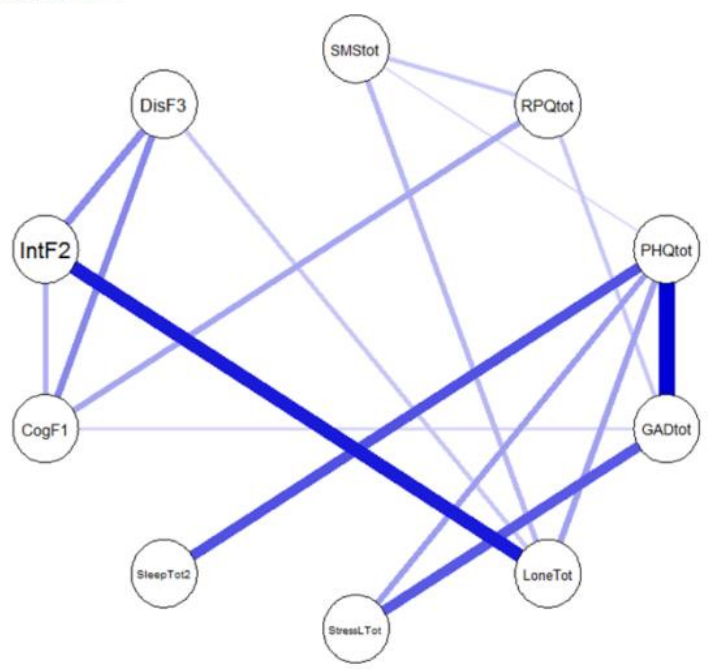

\section{Discussion}

\subsection{Main Findings}

In this three-time point network analysis study of the associations between psychotic-like experiences (paranoia/schizotypal traits) and mental health (anxiety, depression, loneliness, aggression, COVID-related stress, poor sleep), we found that both schizotypal traits and paranoia

452 were positively associated with depression, anxiety, stress, and poor sleep primarily through selfperceived loneliness. Specifically, interpersonal and disorganized features were associated with 
454 loneliness and depression - a key feature in individuals in the high-schizotypy and high-paranoia 455 group but not the low-trait groups - while cognitive-perceptual features of schizotypy were 456 specifically associated with anxiety. Both paranoia/schizotypal traits were uniquely associated 457 with aggression. Interestingly, there were no network structure differences across sex, age 458 groups, countries, and income level, suggesting that no single vulnerable group can be identified. 459 Between time 1 and 2, there was a reduction in schizotypal traits, aggression, but an increase in 460 poor sleep for the same participants. Between time 2 and 3, there was an overall reduction in 461 levels of COVID-related stress, schizotypal traits, aggression, paranoia, and loneliness - likely 462 reflecting the easing of COVID restrictions across countries especially the UK. On balance, these 463 findings suggest that intervening on self-perceived loneliness - an influential variable across all 464 participant groups which may have improved during the easing of lockdown - may break the 465 negative associations between paranoia/schizotypy and negative mental health symptoms, but 466 externalizing symptoms may still remain.

467 Although the empirical evidence for why schizotypal traits is associated with loneliness 468 remains sparse, it is conceivable that individuals with schizotypy often have no close friends, 469 anhedonia, and this in turn may distance other people and result in perceived level of loneliness. 470 Indeed, a large-scale meta-analytic study has documented a moderate effect between loneliness 471 and schizotypal traits $(N=15,647 ; k=13, r=.32,95 \% C I[.20$ - .44]) (Michalska da Rocha, 472 Rhodes, Vasilopoulou, \& Hutton, 2018) that is replicated for both positive and negative 473 symptoms of schizotypy (Badcock et al., 2016). This is also consistent with studies of first474 episode schizophrenia patients who report having more days during the week in which they feel 475 lonely, perhaps associated with the poorer social network and support, and associated symptoms 476 of depression and anxiety (Sündermann et al., 2014). Another explanation for this relationship 477 could be that the fear of others causing harm (paranoia), coupled with an individual's odd 478 behaviors, and social anxiety resulting in avoidance from social situations, can in turn lead to 479 reduced interactions with others, and self-perceived detachment from others (loneliness).

480 Whether this is purely due to the COVID easing of restrictions taking place during time 3 (April 481 to July 2021) or existing poor social support/earlier childhood experiences may be disputed, as 482 we do not have pre-pandemic baseline measures of paranoia. Yet we know from developmental 483 research that compared with trusting children, highly mistrustful 9-16-year-olds were more likely 
484 to report feelings of loneliness, more negative peer relationships like being victims of bullying 485 and a hostile attributional style of thinking about others (Wong, 2015).

486 Over a 12-month period (time 1 and time 3), schizotypal traits and paranoid ideations 487 have reduced over time, and we only see reductions in levels of loneliness between time 2 and 3 $488(p<.002)$ and not between time 1 and time 2 ( $p=.273)$ (see Table 5). Two explanations may 489 account for this: the first is that levels of loneliness were generally felt and sustained for the large 490 majority of people in the sample given that the UK was in full national lockdowns coinciding 491 with time 1 and time 2 and worldwide travel restrictions were in place. By time 3, reductions in 492 self-perceived levels of loneliness were reported coinciding with the initial easing of restrictions, 493 albeit still limited (e.g., reopening of shops and social distancing still in place until the end of 494 time 3 data collection 19 July 2021). Unfortunately, without a fourth time point, it is not possible 495 to see whether levels of loneliness continue to reduce as would be expected to pre-pandemic 496 times. Perhaps unsurprisingly, initial easing with certain restrictions still in place (e.g., limited 497 numbers for gathering, work from home, shops not fully open, vaccine roll-out at 90\%) is 498 helping reduce feelings of loneliness for the majority of respondents. This is consistent with a 499 small experimental study of community samples $(N=60)$ whereby using a false-feedback 500 paradigm to manipulate feelings of loneliness have been shown to lead to decreases in paranoid 501 beliefs (Lamster, Nittel, Rief, Mehl, \& Lincoln, 2017). This suggests that government and 502 community efforts to reduce feelings of loneliness may be beneficial for the majority of the 503 general public.

A second explanation for the evolution of self-perceived levels of loneliness observed in our study is based on individual differences. Participants respond to the survey at different times of the lockdown period, and our assessment at 6/12 months maybe too long to capture smaller inperson fluctuations. Yet we know from our wave 1 findings that the levels of loneliness follow an inverted U-shape to predict the length of lockdown whereby individuals at the beginning and end of the lockdown period reported significantly higher levels of loneliness compared to those in the middle weeks of the lockdown period (Carollo et al., 2021). This may suggest that there

512 and beyond other mental health variables, perhaps relating to an individual's ability to cope and 513 access financial and emotional support during the lockdown period (Fekih-Romdhane, Dissem, 514 \& Cheour, 2021). This was not measures in our study. Thus, future studies using latent class 
515 analysis to identify high vs low levels of loneliness groups in relation to differences in mental

516 health and schizotypal traits may help clarify the role of loneliness in this network.

517 By using network analysis to map out symptoms of paranoia and schizotypy in relation to 518 mental health variables in different groups of individuals (by sex, age, income, country), this 519 study sought to understand which variable(s) may be a key target of intervention for the specific 520 populations - something that prior studies have not investigated. Controlling for other variables 521 in the network, we did not find network structure differences across groups, suggesting that for 522 all groups, loneliness is a key variable through which paranoid ideations and schizotypal traits 523 are associated with heightened levels of mental health issues (e.g., depression, anxiety, poor 524 sleep, covid-related stress). This finding is well-documented in the literature, whereby reductions 525 in loneliness can improve psychological wellbeing for older adults (Chen \& Feeley, 2014) and 526 promising short-term effects of a weekly positive psychology intervention for patients with 527 psychosis (Lim, Penn, Thomas, \& Gleeson, 2019), and community interventions to reduce 528 loneliness as also increased neighbourhood's identification and social belonging (Fong, Cruwys, 529 Robinson, \& Haslam, 2021), and investing in services that prevent social isolation can reduce 530 loneliness as well (Windle, Francis, \& Coomber, 2011).

531 Since most published findings focus primarily on internalizing problems and not 532 externalizing problems - a key gap addressed in this study - the finding that paranoia/schizotypy 533 are uniquely related to aggression highlights the importance of assessing comorbid 534 psychopathology (Wong, Francesconi, \& Flouri, 2021). The schizotypy-aggression relationship 535 observed in this study is consistent with prior pre-pandemic literature (Liu et al., 2019; Wong \& 536 Raine, 2019), indicating that above and beyond the included mental health variables in the 537 network, schizotypal traits are associated with more aggressive behaviors, specifically reactive 538 retaliatory aggression and not proactive, instrumental aggression. This suggests that individuals 539 with high schizotypal traits are unlikely to be individuals who are aggressive toward others, 540 report retaliatory aggression as a result of social interactions with others, and thus more likely to 541 perhaps avoid social situations, engage in reclusive behaviors and report higher feelings of 542 loneliness, despite easing of lockdown that help reduce feelings of loneliness for the majority. 543

\section{4.2. Strengths and Limitations}


This study begins to answer how schizotypal traits and paranoid ideations are associated 546 with various mental health variables for different groups of individuals during the pandemic

547 year. To our knowledge, this is also the first study to explore both internalizing and externalizing 548 problems using a network analytic approach that could likely identify the variable(s) of influence 549 in the network for intervention and demonstrate a holistic mapping of bivariate associations 550 whilst controlling for other network variables. Our study was able to examine macro and micro associations to test for significance across groups and also time points that coincided with

552 national lockdown/easing periods. This analytic technique though not commonly used in 553 behavioural sciences may be valuable when applied to big data in providing a holistic 554 understanding of the web of comorbid relationships that are often observed in mental health 555 research.

This study is not without limitations. First, our participants were recruited online via convenience sampling and may not be generalizable to the population of each country where sample size remained relatively small - although this time-sensitive data may still be helpful where future collaborations with international groups with the same measures are possible. Second, those who chose to take part were particularly willing and had access to technology to complete the survey online, thus potentially they are of a more affluent and motivated group.

562 However, the median income reported by our sample shows that 50\% are under $£ 30,000$ that is 563 similar to the UK National average for 2021, £31,460 (Clark, 2021). Third and finally, our 564 survey relies on self-reporting, which would suggest that the associations between variables are 565 inflated, although arguably self-reporting is the most valid and appropriate method of design given the COVID pandemic restrictions. Nonetheless, these study findings spanning the 12month pandemic period following the same participants do replicate pre-pandemic findings in

568 the literature, specifically highlighting loneliness as a key variable for intervention for governments and local communities in the COVID recovery plans to improve people's

570 psychological and relational health. 


\section{References}

Åkerstedt, T., \& Gillberg, M. (1990). Subjective and objective sleepiness in the active individual. International Journal of Neuroscience, 52(1-2), 29-37.

Bebbington, P. E., McBride, O., Steel, C., Kuipers, E., Radovanoviĉ, M., Brugha, T., ... \& Freeman, D. (2013). The structure of paranoia in the general population. The British Journal of Psychiatry, 202(6), 419-427.

Bortolon, C., Capdevielle, D., Dubreucq, J., \& Raffard, S. (2021). Persecutory ideation and anomalous perceptual experiences in the context of the COVID-19 outbreak in France: what's left one month later?. Journal of Psychiatric Research, 134, 215-222. https://doi.org/10.1016/j.jpsychires.2020.12.042

Borsboom, Denny, and Angélique O. J. Cramer. (2013). Network Analysis: An Integrative Approach to the Structure of Psychopathology. Annual Review of Clinical Psychology 9(1), 91-121. doi: 10.1146/annurev-clinpsy-050212-185608.

Carney, C. E., Buysse, D. J., Ancoli-Israel, S., Edinger, J. D., Krystal, A. D., Lichstein, K. L., Morin, C. M. (2012). The consensus sleep diary: standardizing prospective sleep selfmonitoring. Sleep, 35(2):287-302.

Carollo, A., Bizzego, A., Gabrieli, G., Wong, K. K. Y., Raine, A., \& Esposito, G. (2020). I'm alone but not lonely. U-shaped pattern of perceived loneliness during the COVID-19 pandemic in the UK and Greece. medRxiv. https://doi.org/10.1101/2020.11.26.20239103

Chen, Y., \& Feeley, T. H. (2014). Social support, social strain, loneliness, and well-being among older adults: An analysis of the Health and Retirement Study. Journal of Social and Personal Relationships, 31(2), 141-161.

Clark, D. (2021). Average annual earnings for full-time employees in the UK 1999-2020. https://www.statista.com/statistics/1002964/average-full-time-annual-earnings-in-the-uk/

Daimer, S., Mihatsch, L., Ronan, L., Murray, G. K., \& Knolle, F. (2021). Subjective impact of the COVID-19 pandemic on schizotypy and general mental health in Germany and the UK, for independent samples in May and in October 2020. Frontiers in Psychology, doi: 10.3389/fpsyg.2021.667848 
Dhanani, L. Y., \& Franz, B. (2021). Why public health framing matters: An experimental study of the effects of COVID-19 framing on prejudice and xenophobia in the United States. Social Science \& Medicine, 269, 113572.

Drake, R. J., Pickles, A., Bentall, R. P., Kinderman, P., Haddock, G., Tarrier, N., \& Lewis, S. W. (2004). The evolution of insight, paranoia and depression during early schizophrenia. Psychological Medicine, 34(2), 285-292.

Epskamp, S., Borsboom, D., \& Fried, E. I. (2018). Estimating psychological networks and their accuracy: A tutorial paper. Behavior Research Methods, 50(1), 195-212. https://doi.org/10.3758/s13428-017-0862-1

Fekih-Romdhane, F., Dissem, N., \& Cheour, M. (2021). How did Tunisian university students cope with fear of COVID-19? A comparison across schizotypy features. Personality and Individual Differences, 178, 110872.

Freeman, D., \& Garety, P. A. (2000). Comments on the content of persecutory delusions: does the definition need clarification?. British Journal of Clinical Psychology, 39(4), 407-414.

Freeman, D., Pugh, K., Vorontsova, N., \& Southgate, L. (2009). Insomnia and paranoia. Schizophrenia Research, 108(1-3), 280-284.

Freeman, D., Sheaves, B., Goodwin, G. M., Yu, L. M., Nickless, A., Harrison, P. J., ... \& Espie, C. A. (2017). The effects of improving sleep on mental health (OASIS): a randomised controlled trial with mediation analysis. The Lancet Psychiatry, 4(10), 749-758.

Freeman, D., Stahl, D., McManus, S., Meltzer, H., Brugha, T., Wiles, N., \& Bebbington, P. (2012). Insomnia, worry, anxiety and depression as predictors of the occurrence and persistence of paranoid thinking. Social Psychiatry and Psychiatric Epidemiology, 47(8), 1195-1203.

Freeman, D., Startup, H., Dunn, G., Wingham, G., Černis, E., Evans, N., ... \& Kingdon, D. (2014). Persecutory delusions and psychological well-being. Social Psychiatry and Psychiatric Epidemiology, 49(7), 1045-1050.

Freeman, D., Waite, F., Rosebrock, L., Petit, A., Causier, C., East, A., . . Lambe, S. (2020). Coronavirus conspiracy beliefs, mistrust, and compliance with government guidelines in England. Psychological Medicine, 1-13. doi:10.1017/S0033291720001890 
634 Fong, P., Cruwys, T., Robinson, S. L., Haslam, S. A., Haslam, C., Mance, P. L., \& Fisher, C. L. (2021). Evidence that loneliness can be reduced by a whole-of-community intervention to increase neighbourhood identification. Social Science \& Medicine, 277, 113909.

Foygel, R., \& Drton, M. (2010). Extended Bayesian Information Criteria for Gaussian Graphical Models. Advances in Neural Information Processing Systems, 23, 2020-2028. http://arxiv.org/abs/1011.6640

Ipsos MORI (2020). The UK government's handling of the coronavirus crisis: public perceptions. https://www.kcl.ac.uk/policy-institute/assets/the-handling-of-the-coronaviruscrisis.pdf.

Kroenke, K., Spitzer, R. L., \& Williams, J. B. (2001). The PHQ-9: validity of a brief depression severity measure. Journal of General Internal Medicine, 16(9), 606-613.

Lamster, F., Lincoln, T. M., Nittel, C. M., Rief, W., \& Mehl, S. (2017). The lonely road to paranoia. A path-analytic investigation of loneliness and paranoia. Comprehensive Psychiatry, 74, 35-43. https://doi.org/10.1016/j.comppsych.2016.12.007

Lamster, F., Nittel, C., Rief, W., Mehl, S., \& Lincoln, T. (2017). The impact of loneliness on paranoia: an experimental approach. Journal of Behavior Therapy and Experimental Psychiatry, 54, 51-57.

Lim, M. H., Gleeson, J. F., Alvarez-Jimenez, M., \& Penn, D. L. (2018). Loneliness in psychosis: a systematic review. Social Psychiatry and Psychiatric Epidemiology, 53(3), 221-238. https://doi.org/10.1007/s00127-018-1482-5

Lim, M. H., Penn, D. L., Thomas, N., \& Gleeson, J. F. M. (2020). Is loneliness a feasible treatment target in psychosis?. Social Psychiatry and Psychiatric Epidemiology, 55(7), 901-906.

Liu, J., Wong, K. K. Y., Dong, F., Raine, A., \& Tuvblad, C. (2019). The Schizotypal Personality Questionnaire-Child (SPQ-C): psychometric properties and relations to behavioral problems with multi-informant ratings. Psychiatry Research, 275, 204-211.

Lopes, B., Bortolon, C., \& Jaspal, R. (2020). Paranoia, hallucinations and compulsive buying during the early phase of the COVID-19 outbreak in the United Kingdom: A preliminary experimental study. Psychiatry Research, 293, 113455. https://doi.org/10.1016/j.psychres.2020.113455 
McNally, Richard J. 2021. "Network Analysis of Psychopathology: Controversies and Challenges." Annual Review of Clinical Psychology 17(1):null. doi: 10.1146/annurevclinpsy-081219-092850.

Michalska da Rocha, B., Rhodes, S., Vasilopoulou, E., \& Hutton, P. (2018) Loneliness in Psychosis: A Meta-analytical Review. Schizophrenia Bulletin, 44(1), 114-125, https://doi.org/10.1093/schbul/sbx036

Raine, A., \& Benishay, D. (1995). The SPQ-B: a brief screening instrument for schizotypal personality disorder. Journal of Personality Disorders, 9(4), 346-355.

Raine, A., Dodge, K., Loeber, R., Gatzke-Kopp, L., Lynam, D., Reynolds, C., ... \& Liu, J. (2006). The reactive-proactive aggression questionnaire: Differential correlates of reactive and proactive aggression in adolescent boys. Aggressive Behavior: Official Journal of the International Society for Research on Aggression, 32(2), 159-171.

Russell, D. (1996). UCLA Loneliness Scale (Version 3): Reliability, validity, and factor structure. Journal of Personality Assessment, 66,20-40.

Spitzer, R. L., Kroenke, K., Williams, J. B., \& Löwe, B. (2006). A brief measure for assessing generalized anxiety disorder: the GAD-7. Archives of Internal Medicine, 166(10), 10921097.

Sündermann, O., Onwumere, J., Kane, F. et al. (2014). Social networks and support in firstepisode psychosis: exploring the role of loneliness and anxiety. Social Psychiatry, Psychiatry and Epidemiolology, 49, 359-366. https://doi.org/10.1007/s00127-013-0754-3

Tibshirani, R. (1996). Regression Shrinkage and Selection Via the Lasso. Journal of the Royal Statistical Society: Series B (Methodological), 58(1), 267-288. https://doi.org/10.1111/j.2517-6161.1996.tb02080.x

Tone, E. B., \& Davis, J. S. (2012). Paranoid thinking, suspicion, and risk for aggression: A neurodevelopmental perspective. Development and Psychopathology, 24(3), 1031-1046

Van Borkulo, C., Boschloo, L., Kossakowski, J. J., Tio, P., Schoevers, R., Borsboom, D., \& Waldorp, L. (2017). Comparing network structures on three aspects: A permutation test. Preprint. https://doi.org/10.13140/RG.2.2.29455.38569

Wang, Y., Shi, H. S., Liu, W. H., Zheng, H., Wong, K. K. Y., Cheung, E. F., \& Chan, R. C. (2020). Applying network analysis to investigate the links between dimensional schizotypy and cognitive and affective empathy. Journal of Affective Disorders, 277, 313-321. 
Windle, K., Francis, J., \& Coomber, C. (2011). Preventing loneliness and social isolation: interventions and outcomes (pp. 1-16). London: Social Care Institute for Excellence.

Wong, K. (2020). If COVID-19 is here to stay, how will it affect our mental health and trust in others? https://blogs.ucl.ac.uk/ioe/2020/06/09/if-covid-19-is-here-to-stay-how-will-itaffect-our-mental-health-and-trust-in-others/

Wong, K. K. Y., Francesconi, M., \& Flouri, E. (2021). Internalizing and externalizing problems across childhood and psychotic-like experiences in young-adulthood: The role of developmental period. Schizophrenia Research, 231, 108-114.

Wong, K. K., Freeman, D., \& Hughes, C. (2014). Suspicious young minds: paranoia and mistrust in 8-to 14-year-olds in the UK and Hong Kong. The British Journal of Psychiatry, 205(3), 221-229.

Wong, K. K. \& Raine, A. (2020). COVID-19: Global social trust and mental health study. https://osf.io/fe8q7/

Wong, K. K., Raine, A. (2018). Developmental Aspects of Schizotypy and Suspiciousness: a Review. Current Behavioural Neuroscience Reports, 5, 94-101, https://doi.org/10.1007/s40473-018-0144-y

Wong, K. K., \& Raine, A. (2019). Peer problems and low self-esteem mediate the suspicious and non-suspicious schizotypy-reactive aggression relationship in children and adolescents. Journal of Youth and Adolescence, 48(11), 2241-2254. 
729 Table S1. Correlation coefficients between each pair of variables in network of Wave 2

\begin{tabular}{|c|c|c|c|c|c|c|c|c|c|c|c|}
\hline & $\mathbf{0}$ & 1 & 2 & 3 & 4 & 5 & 6 & 7 & 8 & 9 & 10 \\
\hline 0. SPQ-B total & 1 & & & & & & & & & & \\
\hline 1. SPQ-B Factor1 & $.762 * *$ & 1 & & & & & & & & & \\
\hline 2. SPQ-B Factor2 & $.865 * *$ & $.437 * *$ & 1 & & & & & & & & \\
\hline 3. SPQ-B Factor3 & $.811 * *$ & $.496 * *$ & $.563 * *$ & 1 & & & & & & & \\
\hline 4. SMS & $.424 * *$ & $.380 * *$ & $.323 * *$ & $.348 * *$ & 1 & & & & & & \\
\hline 5. RPQ total & $.160 * *$ & $.218^{* *}$ & 0.059 & $.144 * *$ & $.201 * *$ & 1 & & & & & \\
\hline 6. PHQ total & $.467 * *$ & $.401 * *$ & $.382 * *$ & $.362 * *$ & $.467 * *$ & $.172 * *$ & 1 & & & & \\
\hline 7. GAD total & $.420 * *$ & $.374 * *$ & $.338 * *$ & $.321 * *$ & $.432 * *$ & $.215^{* *}$ & $.789 * *$ & 1 & & & \\
\hline 8. Stress total & $.378 * *$ & $.343 * *$ & $.301 * *$ & $.285^{* *}$ & $.446 * *$ & $.233 * *$ & $.623 * *$ & $.632 * *$ & 1 & & \\
\hline 9. Loneliness total & $.610 * *$ & $.358 * *$ & $.635^{* *}$ & $.450 * *$ & $.487 * *$ & $.150 * *$ & $.569 * *$ & $.514 * *$ & $.453 * *$ & 1 & \\
\hline 10. Sleep total & $.274 * *$ & $.215^{* *}$ & $.235 * *$ & $.218 * *$ & $.256 * *$ & $.082 *$ & $.559 * *$ & $.452 * *$ & $.387 * *$ & $.356 * *$ & 1 \\
\hline
\end{tabular}

730 Note. SPQ-B: Schizotypal Personality Questionnaire - Brief, SMS: Social Mistrust Scale, RPQ:

731 Reactive-Proactive Questionnaire, PHQ: Patient Health Questionnaire-9, GAD: General Anxiety

732 Disorder-7. **: $\mathrm{p}<0.01, *: \mathrm{p}<0.05$. 
736 Table S2. Correlation coefficients between each pair of variables in network of Wave 3

\begin{tabular}{|c|c|c|c|c|c|c|c|c|c|c|c|}
\hline & $\mathbf{0}$ & 1 & 2 & 3 & 4 & 5 & 6 & 7 & 8 & 9 & $\begin{array}{l}\mathbf{1} \\
\mathbf{0}\end{array}$ \\
\hline 0. SPQ-B total & 1 & & & & & & & & & & \\
\hline 1. SPQ-B Factor1 & $.759 * *$ & 1 & & & & & & & & & \\
\hline 2. SPQ-B Factor2 & $.862 * *$ & $.444 * *$ & 1 & & & & & & & & \\
\hline 3. SPQ-B Factor3 & $.780 * *$ & $.470 * *$ & $.499 * *$ & 1 & & & & & & & \\
\hline 4. SMS & $.480 * *$ & $.421 * *$ & $.387 * *$ & $.355^{* *}$ & 1 & & & & & & \\
\hline 5. RPQ total & $.281 * *$ & $.272 * *$ & $.225^{* *}$ & $.186^{* *}$ & $.310 * *$ & 1 & & & & & \\
\hline 6. PHQ total & $.478 * *$ & $.399 * *$ & $.405 * *$ & $.347 * *$ & $.462 * *$ & $.315 * *$ & 1 & & & & \\
\hline 7. GAD total & $.447 * *$ & $.357 * *$ & $.392 * *$ & $.320 * *$ & $.429 * *$ & $.351 * *$ & $.772 * *$ & 1 & & & \\
\hline 8. Stress total & $.408 * *$ & $.397 * *$ & $.323 * *$ & $.270 * *$ & $.428 * *$ & $.319 * *$ & $.633 * *$ & $.610 * *$ & 1 & & \\
\hline 9. Loneliness total & $.636 * *$ & $.408 * *$ & $.653 * *$ & $.414 * *$ & $.556 * *$ & $.289 * *$ & $.609 * *$ & $.517 * *$ & $.480 * *$ & 1 & \\
\hline 10. Sleep total & $.202 * *$ & $.145^{* *}$ & $.185^{* *}$ & $.149 * *$ & $.181 * *$ & $.137 * *$ & $.516 * *$ & $.416 * *$ & $.357 * *$ & $.296 * *$ & 1 \\
\hline
\end{tabular}

737 Note. SPQ-B: Schizotypal Personality Questionnaire - Brief, SMS: Social Mistrust Scale, RPQ:

738 Reactive-Proactive Questionnaire, PHQ: Patient Health Questionnaire-9, GAD: General Anxiety

739 Disorder-7. **: $\mathrm{p}<0.01$. 
Network stability and accuracy

741 Bootstrapping with 2500 permutations was performed to estimate the accuracy of edge-weights.

742 Bootstrapped CIs are plotted in Figure S1. The relatively narrow bootstrapped CIs suggested

743 that the order of the edges in the network was stable.

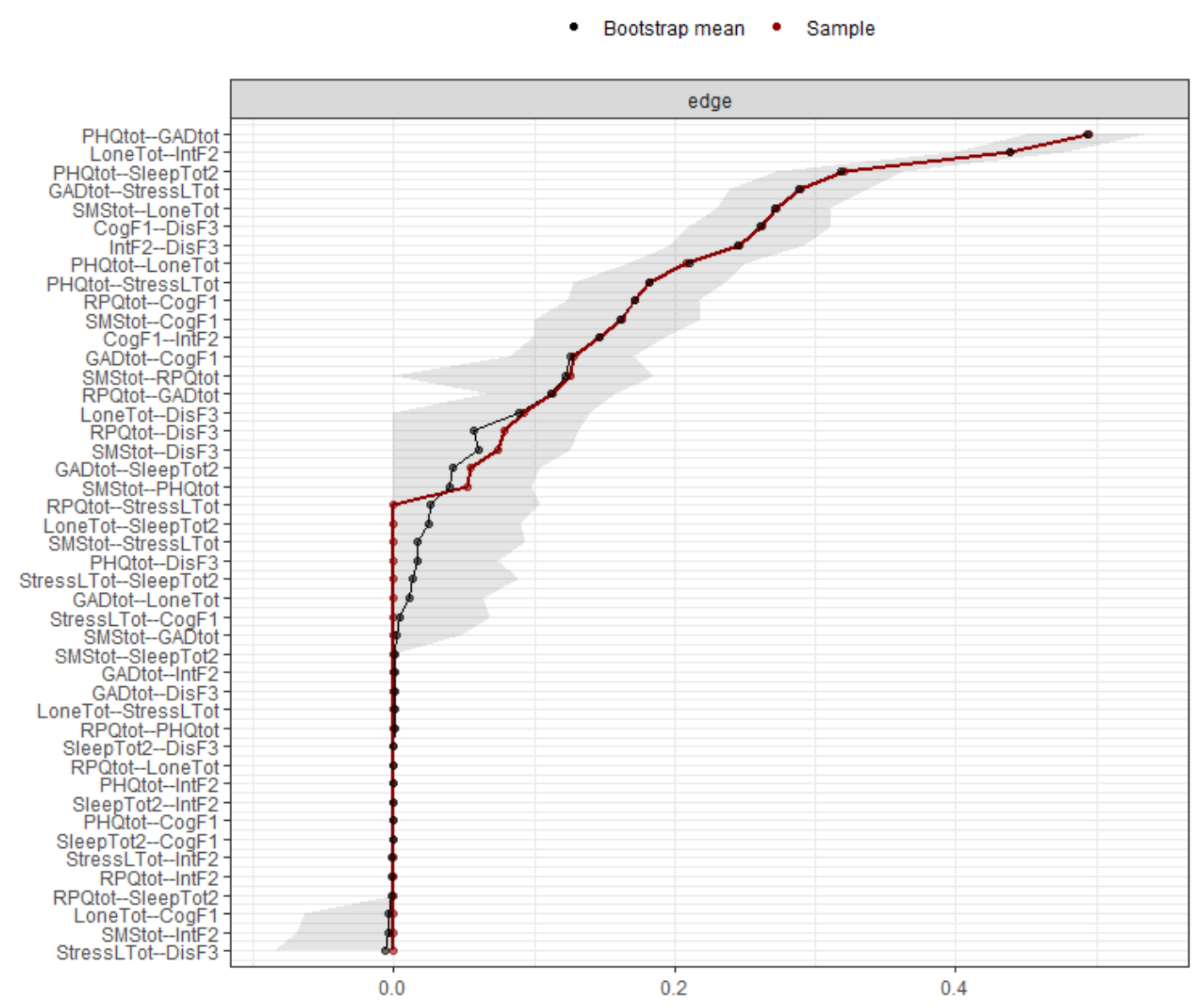

Figure S1. Bootstrapped CIs of estimated edge-weights for the estimated network. The red

746 line indicates the sample values and the grey area indicates the bootstrapped CIs. Each horizontal

747 line represents one edge of the network ordered by edge-weights.

\section{S1.2 Centrality stability}

750 The stability of the order of centrality indices was investigated based on observation of subsets

751 of the data (2500 permutations). Figure S2 below shows the good stability of strength. Stability

752 of centrality indices could be quantified using the CS-coefficient, which calculated the

753 maximum drop in proportions to retain a correlation of 0.7 in at least $95 \%$ of the sample. We 
754 found that the CS-coefficient for strength $(\mathrm{CS}$ (cor=0.7) $=0.75)$ is higher than 0.5 suggesting the 755 centrality indices were stable.

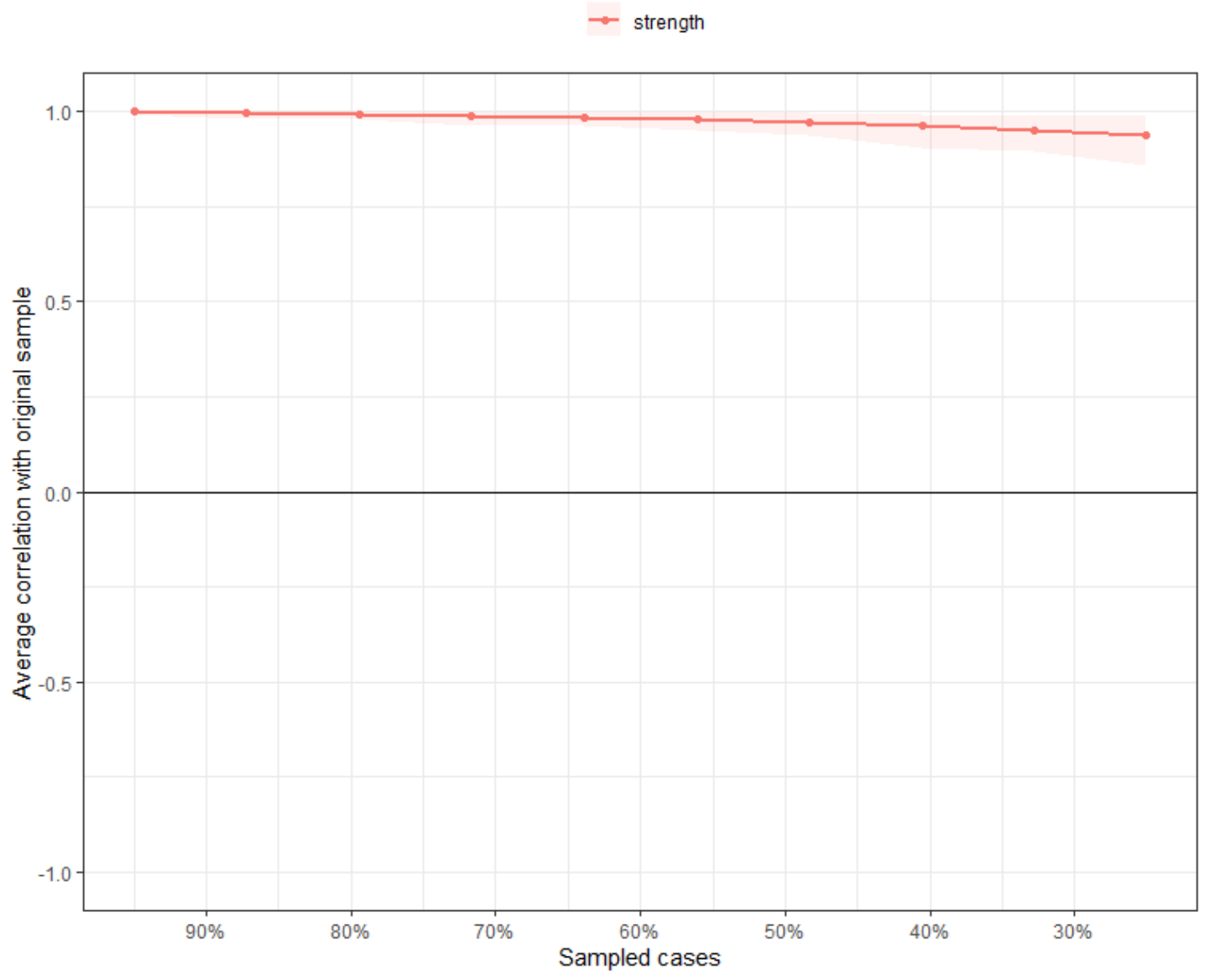

Figure S2. A verage correlations between strengths of networks estimated with sampled

758 participants and original sample. Lines indicate the means and areas indicate the range from

759 the 2.5 th to the 97.5 th percentile.

761 S1.3 Testing for significant differences of edge-weights and centrality

762 We then performed bootstrapped difference tests (with 2500 permutations) of edge-weights and 763 centrality indices to test whether they differed significantly from each other. The results are 764 shown in Figure S3 and S4 respectively. 


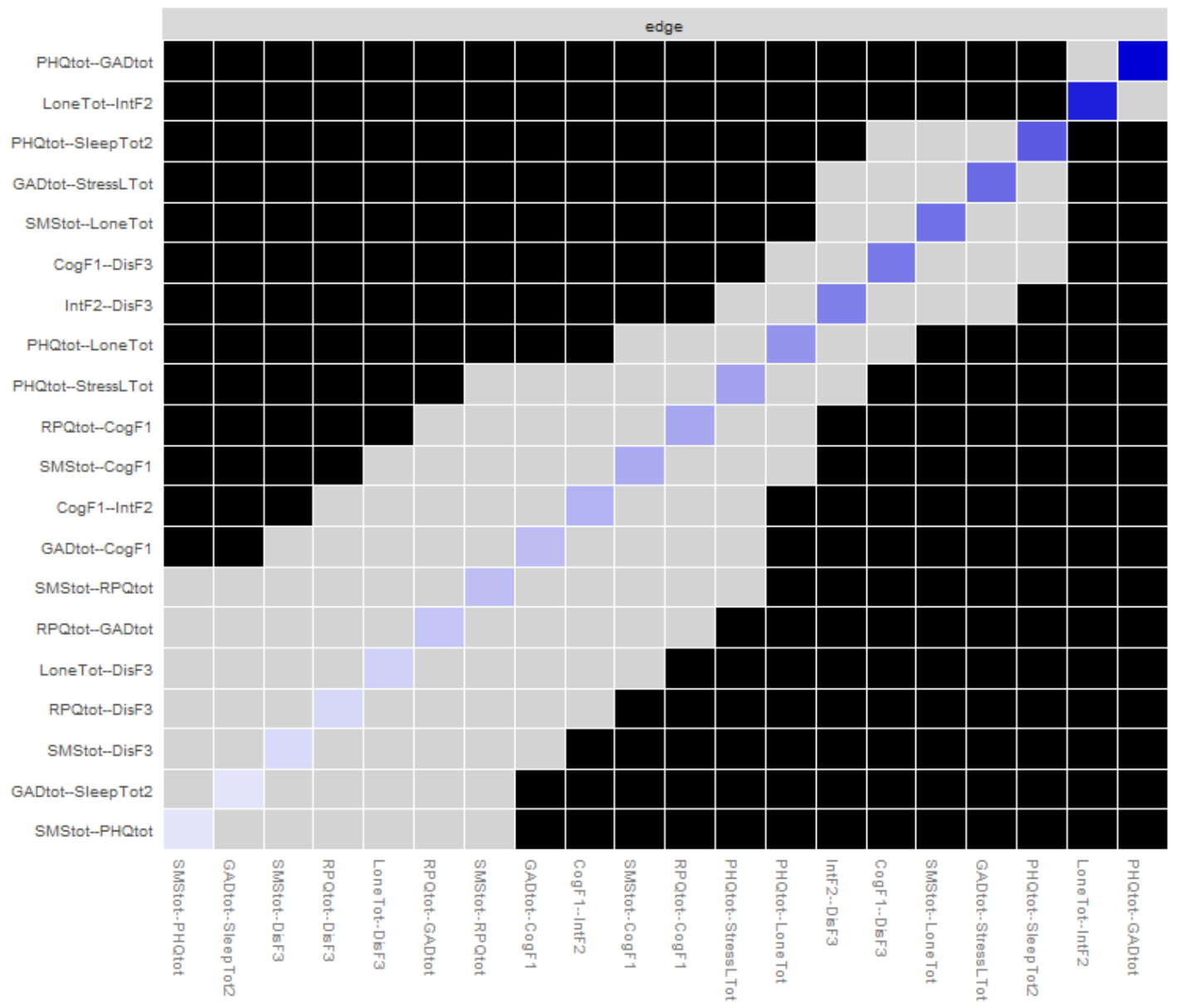

765 
766 Figure S3. Bootstrapped difference tests on the non-zero edge-weights of the estimated network.

767 Black boxes indicate edges that differ significantly from other corresponding edges in the matrix.

768 Coloured boxes in the edge-weight plot correspond to the colour of edges in the estimated 769 network.

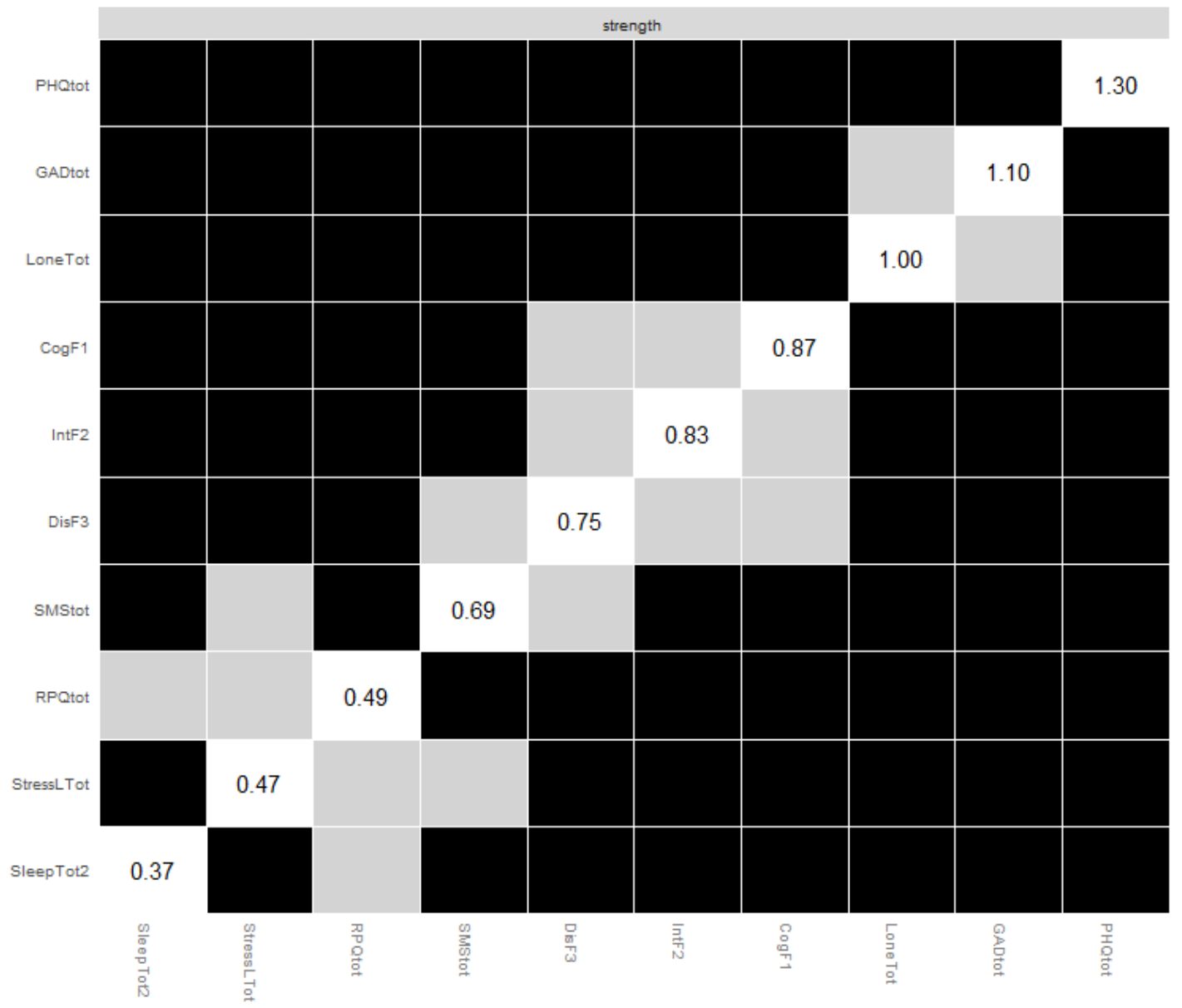


771 Figure S4. Bootstrapped difference tests on the nodal strength of all the variables in the

772 network. Black boxes indicate nodes that differed significantly from another corresponding node

773 in the matrix. Numbers in white boxes in the centrality plot show the strength of the

774 corresponding node.

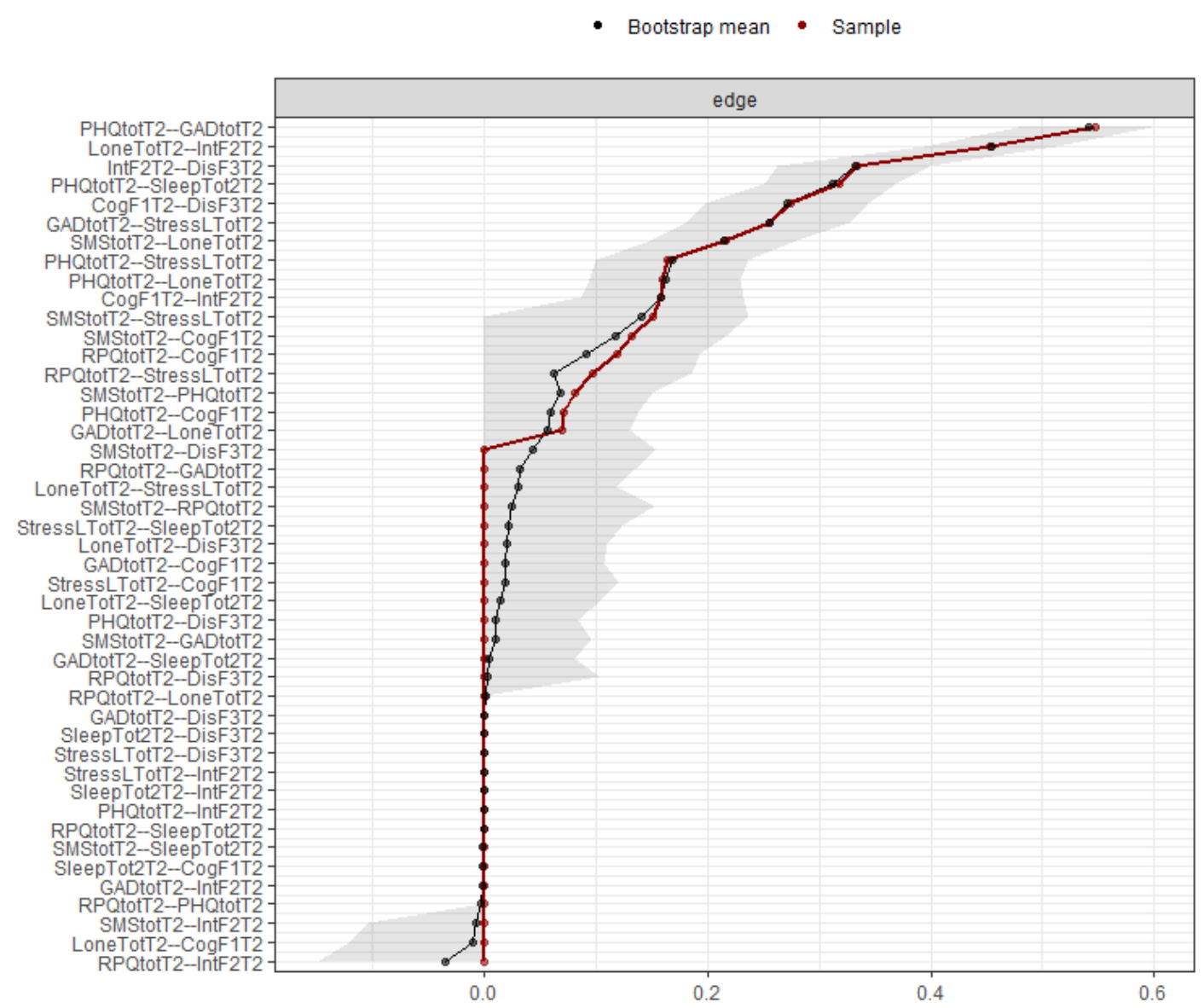

776 Figure S5. Bootstrapped CIs of estimated edge-weights for the estimated network at Wave

777 2. The red line indicates the sample values and the grey area indicates the bootstrapped CIs. Each

778 horizontal line represents one edge of the network ordered by edge-weights. 


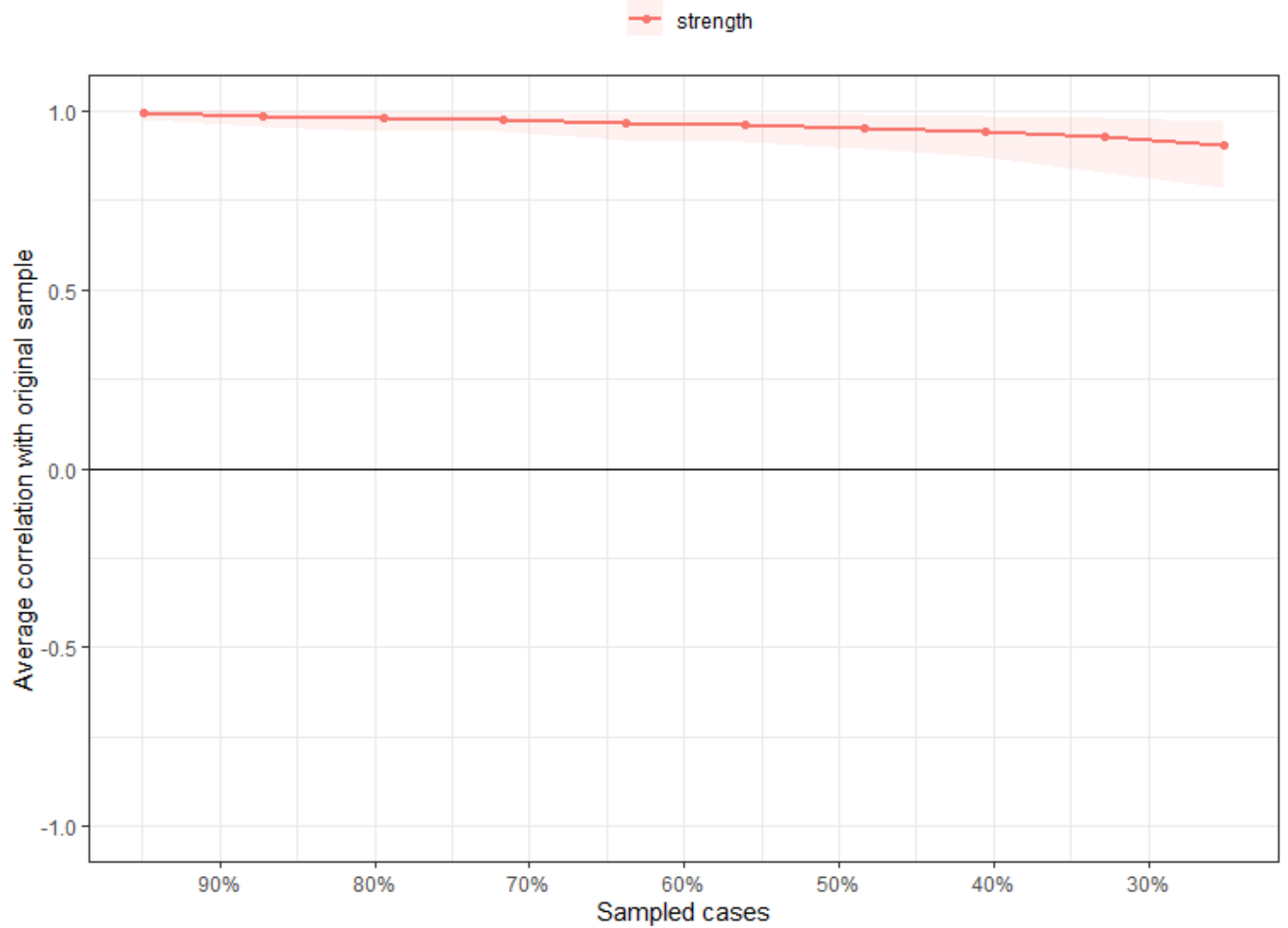

783 Figure S6. Average correlations between strengths of networks estimated for Wave 2 with

784 sampled participants and original sample. Lines indicate the means and areas indicate the 785 range from the 2.5th to the 97.5th percentile. The CS-coefficient for strength (CS (cor $=0.7)=$ $7860.749)$ is higher than 0.5 suggesting the centrality indices were stable. 


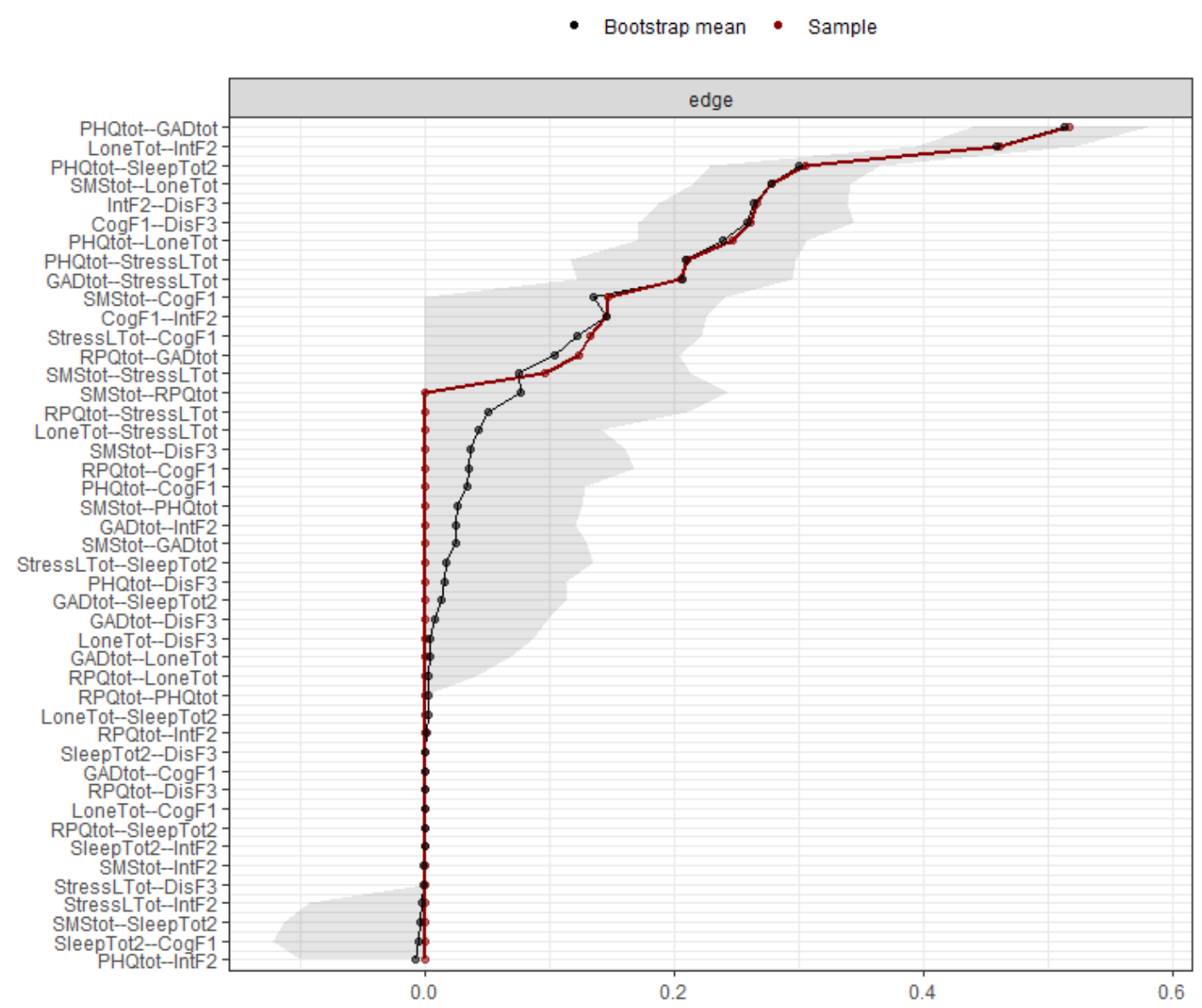

788 Figure S7. Bootstrapped CIs of estimated edge-weights for the estimated network at Wave

789 3. The red line indicates the sample values and the grey area indicates the bootstrapped CIs. Each

790 horizontal line represents one edge of the network ordered by edge-weights. 


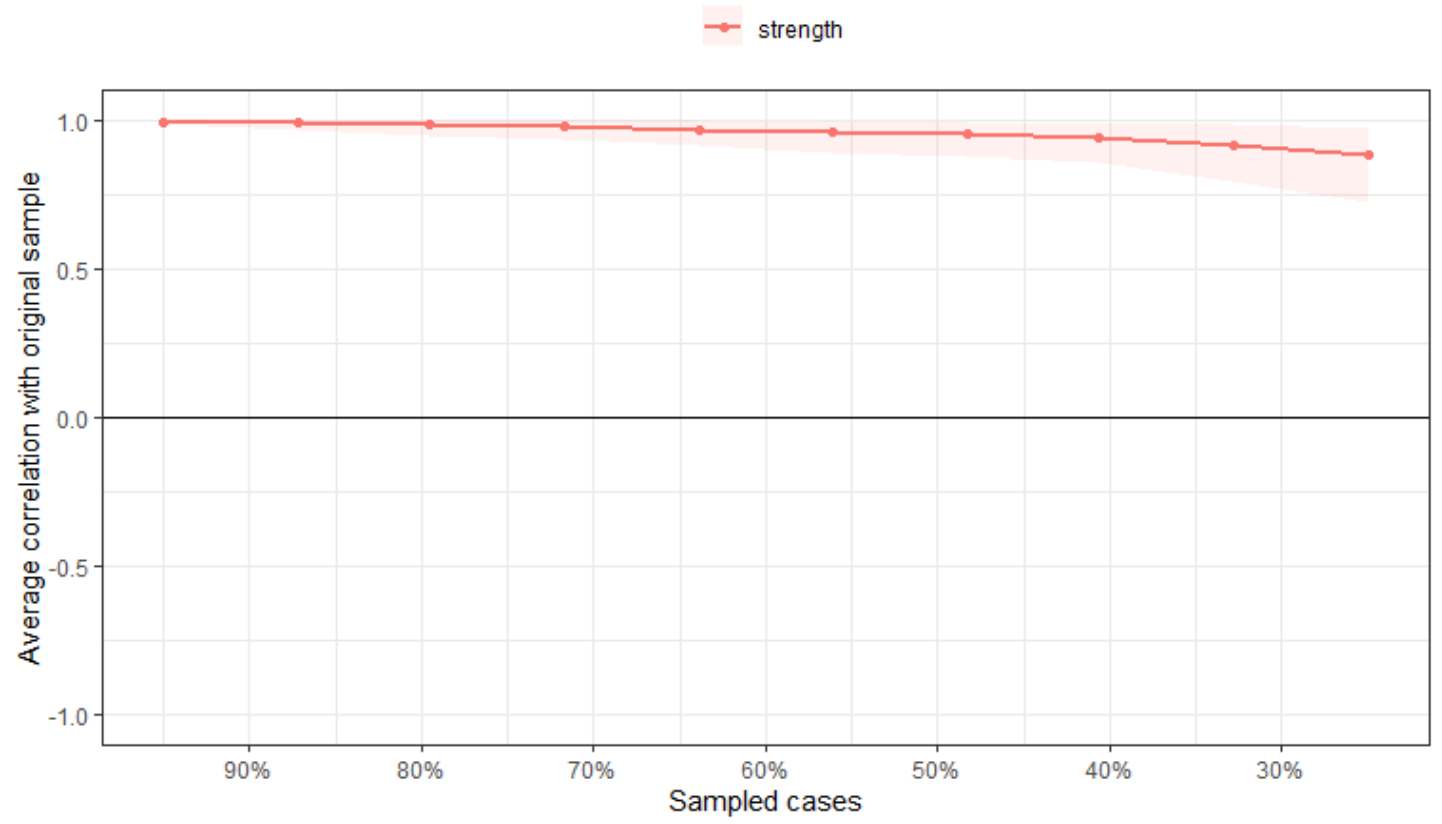

794 sampled participants and original sample. Lines indicate the means and areas indicate the 795 range from the 2.5 th to the 97.5 th percentile. The CS-coefficient for strength $(\mathrm{CS}(\mathrm{cor}=0.7)=$ 796 0.751) is higher than 0.25 suggesting the centrality indices were relatively stable. 